\title{
Failure of Unrestrained Lightly Reinforced Concrete Slabs Under Fire - Part I: Analytical Models
}

\author{
E. Omer ${ }^{1}$, B.A. Izzuddin ${ }^{2}$ and A.Y. Elghazouli ${ }^{3}$
}

\begin{abstract}
Novel models are developed in this paper for the assessment of the failure load of lightly reinforced concrete slabs under fire conditions, considering simply supported rectangular slabs without planar edge restraints. In the limit, this load corresponds to the failure load of composite slabs under fire, since fire tests have demonstrated that the steel deck de-bonds leaving a lightly reinforced concrete slab. The developed models account for the temperature effect on the geometric and material properties, and they consider the tensile membrane action developed at large deflections. The deflected shape, used as the basis of model formulation, was observed experimentally to match the failure mode described by yield line theory, and in the developed models it is assumed that cracks forming along the yield lines penetrate through the slab depth. The strain concentration in the reinforcement along these cracks is established by considering the bond slip characteristics, and the failure load is determined as that corresponding to a specific rupture mechanical reinforcement strain. Comparisons against the non-linear finite element analysis program ADAPTIC are presented. Simplified versions of the proposed models are also presented to assess the failure of composite slabs under elevated temperatures which are shown to correlate favourably with the complete formulations.
\end{abstract}

\footnotetext{
${ }^{1}$ Research student, Dept. Civil \& Env. Eng'g, Imperial College London SW7 2AZ.

${ }^{2}$ Professor of Computational Structural Mechanics, Dept. Civil \& Env. Eng'g, Imperial College London, SW7 2AZ, (Corresponding author, b.izzuddin@imperial.ac.uk), M.ASCE

${ }^{3}$ Reader in Engineering Structures, Dept. Civil \& Env. Eng'g, Imperial College London SW7 2AZ, M.ASCE
} 


\section{Introduction}

Recently, there has been increased interest within the structural engineering community related to the design of structures against fire thanks largely to the advance of computational capabilities [1]-[6]. In this respect, codes such as BS5950-Part:8 [7] have been updated in order to include the effect of fire on the material properties. However, current codes do not yet address the important issue of the actual performance of structural members under elevated temperature, including interaction with the surrounding structure and the influence of thermal expansion and curvature particularly under axial/planar restraint. Important steps towards the understanding of the behaviour of composite slabs have been made, most notably with the fire experiments at the Building Research Establishment's Cardington Large Building Testing Facility. Simplified models [5] for the assessment of the capacity of such slabs under elevated temperature were developed, recognising, though empirically, that failure occurs by rupture of the reinforcement along full depth cracks which form as a result of the large deflections. Nevertheless, the issue of strain concentrations at these cracks was not addressed, which governs failure especially in the context of lightly reinforced concrete members. In such members, which include composite floor slabs, the opening of a crack is not typically followed by the opening of adjacent cracks, since the cracking capacity of the concrete member is typically greater than the ultimate capacity governed by reinforcement strength. In this respect, the strength of the bond between the reinforcement and the surrounding concrete gains importance, since it affects the strain and stress concentrations in the reinforcement at the crack tip. Another important factor is the restraint to horizontal movement existing along the perimeter of the floor slabs. The model developed at BRE [5] assumes that floor slabs are unrestrained horizontally, and that the sides of the slab move in as the loading progresses, which was based on limited experimental observations. The failure mode assumed in that formulation is similar to that predicted by yield-line theory [8], which is supported by fire tests [9], where cracks developed along yield lines and in some cases penetrated the full depth of the slab. Finally, the failure of the slab was assumed to occur by rupture of the reinforcement along the full depth crack, but this was based empirically on average strains obtained from an assumed curved slab configuration. Importantly, this empirical treatment did not account for the important influence of the bond strength on the rupture of the reinforcement. 
A previously developed model captures these effects for a lightly reinforced slab with full-planar restraints along the edges [10]. In the current paper, two model variants for a slab model which is unrestrained against horizontal movement at the supports are presented corresponding to two different experimentally observed failure modes. The proposed slab models consider the development of bond and the resulting stress concentrations in the reinforcement over individual strips, which are taken across the different cracks. Each of these strips are based on similar principles to a newly developed beam model [10], which is a reasonably accurate simplification of a more rigorous previous beam formulation [11].

In the companion paper [12], the kinematic assumptions of the proposed models are verified, and comparisons are made against models previously proposed by other reearchers. The application of the newly developed models and any restrictions are also discussed, and several examples are presented to demonstrate the applicability of these models to the failure assessment of unrestrained slabs under elevated temperature.

\section{Unrestrained Slab Models for Ambient Temperature}

A model for the failure assessment of horizontally restrained LRC slabs under ambient and elevated temperature was developed in [10]. This can be used for the assessment of slabs which are surrounded by stiff boundaries such as in the case of slabs located in the interior of a building. However, if these surroundings are not sufficiently stiff to restrain the edges of the slab from planar movement, or if no surrounding structure exists on certain sides such as in the case of slabs located at the edge of a building, the restrained slab model becomes less applicable, and a new model is required corresponding to planary unrestrained slabs providing a limiting condition which can be used for the assessment of the capacity of such slabs.

Similar to the case of an axially unrestrained beam subject to large deformations (Figure 1), the edges of the slab would pull-in when the out-of-plane deformations are sufficiently large. For slabs, this inward movement results in a crack, additional to the yield line cracks, occuring perpendicular to the longer edge running across the short span and penetrating the depth of the slab [13]. The slab parts bounded by the yield lines and the additional crack rotate about an axis perpendicular to the slab surface. However, the exact location of this additional crack can not be determined, since from a number of experiments [13]-[15] it was observed that the crack can 
occur at either the middle of the long span or at the intersection of the diagonal yield lines with the central yield line (Figure 2).

A model was previously developed at the Building Research Establishment for the failure assessment of LRC slabs under elevated temperatures [16], which was based on the assumption that the crack along the short span occurs at the centre of the long direction (Figure 2(a)). The model makes certain assumptions about the distribution of internal stresses, and the loaddeflection response is obtained by satisfying equilibrium of the slab parts, including the effect of out-of-plane displacements. The failure is based on a semi-empirical formulation, where it is assumed that the slab fails when the average strain of the reinforcement at the centre of the slab running in the long direction reaches a specific value, which is calibrated so that the failure displacement predicted by the model remains below the experimentally observed displacements. However, this empirical treatment is far removed from sound engineering principles, and cannot account for such important factors as the bond strength and the reinforcement stress-strain response.

In this paper, the model developed previously for the failure assessment of restrained slabs [10] is modified in order to include the planar movement of the slab edges. This movement affects the crack widths and consequently the forces in the reinforcement. Two variants of the model are presented, with the first corresponding to the case of a full-depth crack at the centre of the slab (Figure 2(a)), denoted hereafter as CM, and the second corresponding to the case of two full-depth cracks at the intersection of the yield lines (Figure 2(b)), denoted as IM. The full formulation for the CM model is included here, and the reader is referred to [17] for the full formulation of the similar IM model, the derivation of which is presented here in less detail than the CM model. The kinematic assumptions of the two variant models will be validated, and comparisons against the current BRE method and available experimental data will be made in the companion paper [12].

As mentioned above, the experimental studies do not provide a clear indication about the location of the full depth crack across the short span or the parameters affecting this location, thus the need to develop the two CM and IM models. The assumptions used for the two models are similar to those made and verified for the previous restrained slab model [10], and can be summarised as: 
- the slab has a single layer of reinforcement which is anchored at the supports;

- the reinforcement in the two directions is assumed to be at the same level;

- the slab is simply supported along the edges;

- rigid-hardening material model is adopted for steel;

- the bond-slip response is described by a rigid-plastic law, and concrete is rigid in compression with negligible tensile strength;

- cracks are only formed at the locations given by the yield line theory and across the short span; either at the centre of the slab (CM model) or at the intersection of the yield lines (IM model), as shown in Figure 2;

- the various parts of the slab, bounded by the yield lines and through-depth cracks, are simply supported along the edges and are free to rotate both in-plane and out-of-plane in a rigid manner;

- for the CM model (Figure 2(a)), the triangular and trapezoidal parts are in contact at the slab corners at the top fibre; in the short direction at midspan, the trapezoidal parts are in contact at the top fibre; in the long direction along the edge support, the trapezoidal part is in contact with the adjacent trapezoidal part;

- for the IM model (Figure 2(b)), the triangular parts are in contact at the slab corner at the top fibre; in the short direction, the rectangular parts are in contact at the top fibre along the full length of the central yield line; in the long direction along the edge support, the triangular and rectangular parts are in contact; and

- failure occurs by rupture of the reinforcement across the through-depth cracks.

Based on the above, the slab fails by rotating rigidly with respect to the yield lines. According to the yield line theory, an isotropic slab supported on all four edges and loaded uniformly develops cracks at yield lines defined by the parameter $\eta$, which referring to Figure 3 is a function of the aspect ratio $\alpha$ of the slab and is given by [8]:

$$
\begin{aligned}
& \alpha=\frac{a}{b} \ldots \ldots \ldots \ldots \ldots \ldots \ldots \ldots \ldots \ldots \ldots \ldots \\
& \eta
\end{aligned}
$$


Moreover, referring to Figure 4, where the concrete cover to the reinforcement is not shown, for a restrained beam with length of $2 L$ and midspan point load of $2 P$, the extension $\Delta_{s}$ of the reinforcement on one side of the midspan can be obtained as [10]:

$$
\Delta_{s}=L-\sqrt{L^{2}-U^{2}}
$$

where $U$ is the midspan deflection.

As a result of the rigid-hardening steel assumption, this extension will be due to the extension of the reinforcement in a region where the steel stress exceeds yield. Assuming that the member is sufficiently long, the force drops from $T_{s}$ at the crack face, according to the bond strength per unit length $\sigma_{b}$, to the force corresponding to the yield strength of the steel $T_{y}$ at a distance $l_{b}$ from the crack face (Figure 5), defined as the bond length. However, if the length of the member is not sufficient to have the steel stress reaching the yield strength the bond length $l_{b}$ would be limited to the member length.

Therefore $l_{b}$ can be expressed as:

$$
l_{b}=\min \left(\frac{T_{s}-T_{y}}{\sigma_{b}}, L\right)
$$

Considering that at a distance $x$ from the crack face, the force $T(x)$ and the strain $\varepsilon_{s}$ in the reinforcement are respectively given by:

$$
\begin{aligned}
& T(x)=T_{s}-\sigma_{b} x \\
& \varepsilon_{s}=\left\{\begin{array}{lll}
\frac{T(x)-T_{y}}{A_{s} E_{2}} & x<l_{b} 0 \quad x \geq l_{b}
\end{array}\right.
\end{aligned}
$$

the extension of the reinforcement $\Delta_{s}$ is obtained from: 


$$
\Delta_{s}=\int_{0}^{l} b \varepsilon_{s} \mathrm{~d} x
$$

as:

$$
\Delta_{s}= \begin{cases}\frac{\left(T_{s}-T_{y}\right)^{2}}{2 A_{s} E_{2} \sigma_{b}} & \text { if } \quad l_{b} \leq L \\ \frac{\left(T_{s}-T_{y}\right) L}{A_{s} E_{2}}-\frac{\sigma_{b} L^{2}}{2 A_{s} E_{2}} & \text { if } \quad l_{b}=L\end{cases}
$$

where $A_{s}$ is the area of the reinforcement and $E_{2}$ is the hardening modulus of steel (Figure 6). Therefore, the force in the reinforcement at the crack face is obtained in terms of $\Delta_{s}$ as:

$$
T_{s}=\left\{\begin{array}{lll}
T_{y}+\sqrt{2 A_{s} E_{2} \sigma_{b} \Delta_{s}} & \text { if } & l_{b} \leq L \\
T_{y}+\frac{\sigma_{b} L}{2}+\frac{A_{s} E_{2} \Delta_{s}}{L} & \text { if } & l_{b}=L
\end{array}\right.
$$

Combining Equations (3) and (9), the rate of energy dissipated by the reinforcement and bond-slip $\dot{D}_{s}$ can now be obtained in terms of $U$ as:

$$
\dot{D}_{s}=T_{s} \frac{\mathrm{d} \Delta_{s}}{\mathrm{~d} U}
$$

This beam analogy is extended here to represent the behaviour of unrestrained slabs with appropriate modifications, noting that the energy dissipated by concrete can be neglected due to the assumption of rigid compressive behaviour and no tensile strength. Due to symmetry, only a quarter of the slab is considered and strips across the two directions of the slab are treated as LRC beams. Two models are developed and the essentials of derivation of the CM model are presented, with only a summary of the important attributes for the IM model given. 


\subsection{Centre Crack Model}

As mentioned before, for the CM model, the triangular and the trapezoidal parts are in contact at the top fibre at the slab corner. In line with the rigid concrete assumption, this causes the slab parts to rotate with respect to axes passing through the top fibers of the slab at the support as the slab part deflects, resulting in an outwards movement of the slab at the level of reinforcement at the corner, as depicted in Figure 7.

With increasing deflections, the trapezoidal part rotates in-plane so as to remain in contact with the adjacent trapezoidal part at the top fibre at the centre of the slab (Figure 7). The planar rotation of the trapezoidal part $\phi_{c}$ can be obtained with reference to Figures 7 and 8(a) as:

$$
\phi_{c}=\frac{b}{a}-\sqrt{\frac{b^{2}}{a^{2}}-\frac{4 U_{c}^{2}}{a^{2}}}
$$

\subsubsection{Strip across the central yield line}

The extension of the reinforcement across the central yield (Figure 8) line at the midpoint of the slab $\Delta_{s c \mid x=a / 2}^{c}$ is given by:

$$
\Delta_{s c \mid x=a / 2}^{c}=\frac{2 d U_{c}}{b}
$$

where the superscript $c$ refers to the CM model.

The extension of the reinforcement across the central yield line increases towards the intersection of the yield lines linearly due to the planar rotation (Figure 7) and is given as:

$$
\Delta_{s c e}^{c}=\frac{2 d U_{c}}{b}+\phi_{c}\left(\frac{a}{2}-x\right)
$$

The force in the reinforcement across the central yield line can be obtained from (9) as: 


$$
T_{s c e}^{c}=\left\{\begin{array}{lll}
T_{y}+\sqrt{2 A_{s} E_{2} \sigma_{b} \Delta_{s c e}^{c}} & \text { if } \sqrt{\frac{2 A_{s} E_{2} \Delta_{s c e}^{c}}{\sigma_{b}}} \leq \frac{b}{2} \\
T_{y}+\frac{\sigma_{b} b}{4}+\frac{2 A_{s} E_{2} \Delta_{s c e}^{c}}{b} & \text { if } \sqrt{\frac{2 A_{s} E_{2} \Delta_{s c e}^{c}}{\sigma_{b}}} \geq \frac{b}{2}
\end{array}\right.
$$

The rate of energy dissipated across the central yield line is therefore given by:

$$
\dot{D}_{s c e}^{c}=\int_{\eta a}^{a / 2} T_{s c e}^{c} \frac{\mathrm{d} \Delta_{s c e}^{c}}{\mathrm{~d} U_{c}} \mathrm{~d} x
$$

\subsubsection{Strip across the diagonal yield line in the $y$-direction}

The extension of the reinforcement across the diagonal yield line in the $y$-direction can be obtained from the corresponding expression of the restrained slab case [10]:

$$
\Delta_{\text {sdy }}=\frac{x}{\eta a}\left(\frac{b}{2}-\sqrt{\frac{b^{2}}{4}-U_{c}^{2}}\right)
$$

by i) adding the additional extension due to the outwards movement of the slab at the reinforcement level at the corner of the slab, and ii) subtracting the inwards movement of the trapezoidal slab part due to the in-plane rotation, thus resulting in:

$$
\Delta_{s d y}^{c}=\frac{x}{\eta a}\left(\frac{b}{2}-\sqrt{\frac{b^{2}}{4}-U_{c}^{2}}\right)-\phi_{c} x+\frac{2 d U_{c}}{b} \Rightarrow \Delta_{s d y}^{c}=\frac{1-2 \eta}{2 \eta} \phi_{c} x+\frac{2 d U_{c}}{b}
$$

Following the same procedure that was adopted for the restrained slab case [10], the force in the reinforcement in the region adjacent the corner of the slab, the middle of the diagonal yield line and in the region adjacent to the intersection of the yield lines is given respectively by: 


$$
\begin{aligned}
& T_{s y 1}^{c}=T_{y}-\sigma_{b} \frac{b x}{2 \eta a}+\sqrt{2 \sigma_{b}^{2}\left(\frac{b x}{2 \eta a}\right)^{2}+2 \sigma_{b} A_{s} E_{2} \Delta_{s d y}^{c}} \\
& T_{s y 2}^{c}=T_{y}+\sqrt{A_{s} E_{2} \sigma_{b} \Delta_{s d y}^{c}} \\
& T_{s y 3}^{c}=T_{y}-\sigma_{b}\left(\frac{b}{2}-\frac{b x}{2 \eta a}\right)+\sqrt{2 \sigma_{b}^{2}\left(\frac{b}{2}-\frac{b x}{2 \eta a}\right)^{2}+2 \sigma_{b} A_{s} E_{2} \Delta_{s d y}^{c}}
\end{aligned}
$$

where the limits of the regions are obtained as:

$$
\begin{aligned}
& X_{1}^{c}=\frac{\eta a^{2} A_{s} E_{2}}{\sigma_{b} b^{2}}(1-2 \eta) \phi_{c}\left[1+\sqrt{1+\frac{8 \sigma_{b} b d U_{c}}{A_{s} E_{2} a^{2} \phi_{c}^{2}(1-2 \eta)^{2}}}\right] \\
& X_{2}^{c}=\eta a\left(1+\frac{A_{s} E_{2}(1-2 \eta) a \phi_{c}}{\sigma_{b} b^{2}}\right)\left[1-\sqrt{1+\frac{8 A_{s} E_{2} \sigma_{b} b d U_{c}-\sigma_{b}^{2} b^{4}}{\left(\sigma_{b} b^{2}+A_{s} E_{2} a \phi_{c}(1-2 \eta)\right)^{2}}}\right]
\end{aligned}
$$

Thus, the force in the reinforcement crossing the diagonal yield line in the $y$-direction is given by:

$$
T_{s y}^{c}=\left\{\begin{array}{lll}
T_{s y 1}^{c} & \text { for } & 0<x \leq X_{1}^{c} \\
T_{s y 2}^{c} & \text { for } & X_{1}^{c} \leq x \leq X_{2}^{c} \\
T_{s y 3}^{c} & \text { for } & X_{2}^{c} \leq x \leq \eta a
\end{array}\right.
$$

Equation (23) is valid provided that $X_{2}^{c} \geq X_{1}^{c}$ which can be expressed as:

$$
X_{2}^{c} \geq X_{1}^{c} \Rightarrow \phi_{c} \leq \frac{\sigma_{b} b^{3}-32 A_{s} E_{2} d U_{c}}{4 a b A_{s} E_{2}(1-2 \eta)}
$$

It should be noted that $\phi_{c}$ also depends on $U_{c}$, and hence the above expression provides a corresponding limit on $U_{c}$ which can be easily established. As mentioned in the restrained slab 
case for typical slab and bond strength configurations [10], the above condition is satisfied until the point of failure. If on the other hand it is violated, the formulations must be modified to accomodate this effect.

The rate of energy dissipated by the reinforcement crossing the diagonal yield line in the $y$-direction is therefore given by:

$$
\dot{D}_{s d y}^{c}=\int_{0}^{\eta a} T_{s y}^{c} \frac{\mathrm{d} \Delta_{s d y}^{c}}{\mathrm{~d} U_{c}} \mathrm{~d} x
$$

\subsubsection{Strip across the diagonal yield line in the $x$-direction}

Similar to the previous case, the extension of the reinforcement across the diagonal yield line in the $x$-direction is obtained from the corresponding restrained slab case [10]:

$$
\Delta_{s d x}=\frac{2 y}{b}\left(\eta a-\sqrt{\eta^{2} a^{2}-U_{c}^{2}}\right)=\frac{2 y}{b} \Delta_{s c x}
$$

by i) subtracting the inwards movement of the trapezoidal part and ii) adding the outwards movement of the triangular part due to the contact at the top fibre of the slab corner leading to:

$$
\Delta_{s d x}^{c}=\frac{2 y}{b}\left(\eta a-\sqrt{\eta^{2} a^{2}-U_{c}^{2}}\right)-\phi_{c} y+\frac{d U_{c}}{\eta a}
$$

As in the restrained slab case [10], the bond lengths are limited in the regions adjacent to the ends of the diagonal line. The forces near the corner, the middle of the diagonal and near the intersection of the yield lines are respectively given by:

$$
T_{s x 1}^{c}=T_{y}-\sigma_{b} \frac{2 \eta a y}{b}+\sqrt{2 \sigma_{b}^{2}\left(\frac{2 \eta a y}{b}\right)^{2}+2 \sigma_{b} A_{s} E_{2} \Delta_{s d x}^{c}}
$$




$$
\begin{aligned}
& T_{s x 2}^{c}=T_{y}+\sqrt{A_{s} E_{2} \sigma_{b} \Delta_{s d x}^{c}} \\
& T_{s \times 3}^{c}=T_{y}-\sigma_{b}\left(\frac{a}{4}-\frac{\eta a y}{b}\right)+\sqrt{2 \sigma_{b}^{2}\left(\frac{a}{4}-\frac{\eta a y}{b}\right)^{2}+2 \sigma_{b} A_{s} E_{2} \Delta_{s d x}^{c}}
\end{aligned}
$$

The extents of the regions for the above forces in the reinforcement are affected by the fact that there are two cracks along the short span limiting the bond length of the bars. For simplicity, it is assumed that the reinforcement is anchored at the line bisecting the central yield line of the quarter slab $\left(y=b / 2, x=\frac{a}{4}(1-2 \eta)\right)$ and the support of the quarter slab ( $y=0, x=a / 4$ ) as depicted in Figure 9. However, this results in a discrepancy for slabs tending to square where according to this model two distinct cracks and hence two distinct forces exist at the centre of the slab when there is actually a single crack equal to the sum of the full depth crack and the diagonal yield line crack. For the case of a square slab the energy dissipated at that point according to the current formulation is :

$$
\left(T_{y}+\sqrt{2 A_{s} E_{2} \sigma_{b} \Delta_{s d x}^{c}}\right) \dot{\Delta}_{s d x}^{c}+\left(T_{y}+\sqrt{2 A_{s} E_{2} \sigma_{b} \Delta_{s c r}^{c}}\right) \dot{\Delta}_{s c r}^{c}
$$

whereas the real energy dissipation is:

$$
\left(T_{y}+\sqrt{2 A_{s} E_{2} \sigma_{b}\left(\Delta_{s d x}^{c}+\Delta_{s c r}^{c}\right)}\right)\left(\dot{\Delta}_{s d x}^{c}+\dot{\Delta}_{s c r}^{c}\right)
$$

From the above it can be observed that the current formulation predicts less energy dissipation than in reality and hence it provides a conservative approach. Nevertheless, the difference between the two cases is not significant and hence the approach given here can be safely employed.

The limits of the regions $Y_{1}^{c}$ and $Y_{2}^{c}$ can be obtained from considering the bond length:

$$
l_{b}=\frac{T_{s \times 2}^{c}-T_{y}}{\sigma_{b}}
$$


and setting $l_{b}$ equal to the length of the short segments $\frac{2 \eta a y}{b}$ and $\frac{a}{4}-\frac{\eta a y}{b}$, respectively, leading to:

$$
\begin{aligned}
& Y_{1}^{c}=\frac{A_{s} E_{2} b}{8 \eta^{2} a^{2} \sigma_{b}}\left[2\left(\eta a-\sqrt{\eta^{2} a^{2}-U_{c}^{2}}\right)-\phi_{c} b\right] \times \\
&\left\{1+\sqrt{1+\frac{16 \eta a \sigma_{b} d U_{c}}{A_{s} E_{2}\left[2\left(\eta a-\sqrt{\eta^{2} a^{2}-U_{c}^{2}}\right)-\phi_{c} b\right]^{2}}}\right\} \cdots \cdots \cdots . . \cdots \cdots \\
& Y_{2}^{c}=\left\{\frac{b}{4 \eta}+\frac{A_{s} E_{2} b}{2 \eta^{2} a^{2} \sigma_{b}}\left[2\left(\eta a-\sqrt{\eta^{2} a^{2}-U_{c}^{2}}\right)-\phi_{c} b\right]\right\} \times \\
&\left\{1-\sqrt{1+\frac{16 A E \eta a \sigma_{b} b^{2} d U_{c}-\eta^{2} a^{4} b^{2} \sigma_{b}^{2}}{\left[\eta a^{2} \sigma_{b} b+2 A_{s} E_{2} b\left[2\left(\eta a-\sqrt{\eta^{2} a^{2}-U_{c}^{2}}\right)-\phi_{c} b\right]\right]^{2}}}\right\}
\end{aligned}
$$

However, $Y_{2}^{c}$ is valid only after a certain deflection, since in the initial stages of the loading the bond length is not limited. This deflection can be obtained by solving the following equation:

$$
\frac{T_{s \times 2}^{c}-T_{y}}{\sigma_{b}}=\frac{a}{4}(1-2 \eta) \text { at }\left(y=\frac{b}{2}\right)
$$

By using a simplification of the type:

$$
\sqrt{1+u} \approx 1+\frac{u}{2}
$$

the limiting deflection is obtained as: 


$$
U_{c 1}^{c}=\frac{d}{1-2 \eta}\left[\sqrt{1+\frac{\sigma_{b} \eta a^{3}(1-2 \eta)^{3}}{8 A_{s} E_{2} d^{2}}}-1\right]
$$

Thus, the force in the reinforcement across the diagonal yield line in the $x$-direction is given by:

$$
T_{s x}^{c}=\left\{\begin{array}{l}
\left\{\begin{array}{lll}
T_{s \times 1}^{c} & \text { for } & 0<y \leq Y_{1}^{c} \\
T_{s \times 2}^{c} & \text { for } & Y_{1}^{c}<y \leq \frac{b}{2}
\end{array} \text { if } \quad 0<U_{c} \leq U_{c 1}^{c}\right.
\end{array}\right\}
$$

Similar to the $y$-direction, Equation (37) is applicable while the following condition holds:

$$
\frac{b}{2} \geq Y_{2}^{c} \geq Y_{1}^{c}
$$

which is the case for typical slab configurations, though if not the above formulations should be adjusted accordingly.

Considering the above, the rate of energy dissipated in the reinforcement crossing the diagonal yield line in the $x$-direction is given by:

$$
\dot{D}_{s d x}^{c}=\int_{0}^{b / 2} T_{s x}^{c} \frac{\mathrm{d} \Delta_{s d x}^{c}}{d U_{c}} \mathrm{~d} y
$$

\subsubsection{Strip across the full depth crack}

The extension of the reinforcement across the full-depth crack running perpendicular to 
the long span increases linearly towards the mid-point of the slab as given by:

$$
\Delta_{s c r}^{c}=\phi_{c} y
$$

Similar to the previous cases, the force in the reinforcement crossing the full depth crack is described by two regions: one adjacent to the edge of the slab where the bond length is not limited and one adjacent to the centre of the slab where the bond length is limited after a certain deflection. The forces in these regions are respectively given by:

$$
\begin{aligned}
& T_{s c r 1}^{c}=T_{y}+\sqrt{2 A_{s} E_{2} \sigma_{b} \Delta_{s c r}^{c}} \\
& T_{s c r 2}^{c}=T_{y}-\sigma_{b}\left(\frac{a}{4}-\frac{\eta a y}{b}\right)+\sqrt{2 \sigma_{b}^{2}\left(\frac{a}{4}-\frac{\eta a y}{b}\right)^{2}+2 \sigma_{b} A_{s} E_{2} \Delta_{s c r}^{c}}
\end{aligned}
$$

The limit between the regions is obtained by solving:

$$
\frac{T_{s c r 1}^{c}-T_{y}}{\sigma_{b}}=\frac{a}{4}-\frac{\eta a y}{b}
$$

as:

$$
Y_{c}^{c}=\left(\frac{b}{4 \eta}+\frac{A_{s} E_{2} \phi_{c} b^{2}}{\sigma_{b} \eta^{2} a^{2}}\right)\left[1-\sqrt{1-\frac{\sigma_{b}^{2} \eta^{2} a^{4}}{\left(\sigma_{b} \eta a^{2}+4 A_{s} E_{2} \phi_{c} b\right)^{2}}}\right]
$$

As before, this limit is valid after the bond length in the $x$-direction at the centre of the slab becomes limited. The deflection corresponding to this point is similarly obtained as:

$$
U_{c 2}^{c}=\sqrt{\frac{b^{2}}{4}-\left[\frac{b}{2}-\frac{\sigma_{b} a^{3}}{32 A_{s} E_{2} b}(1-2 \eta)^{2}\right]^{2}}
$$


Thus, the force in the reinforcement across the full depth crack is given by:

$$
T_{s c r}^{c}=\left\{\begin{array}{lll}
\left\{\begin{array}{lll}
T_{s c r 1}^{c} & \text { for } & 0<y \leq \frac{b}{2}
\end{array} \quad\right. \text { if } & 0<U_{c} \leq U_{c 2}^{c} \\
\left\{\begin{array}{lll}
T_{s c r 1}^{c} & \text { for } & 0<y \leq Y_{c}^{c} \\
T_{s c r 2}^{c} & \text { for } & Y_{c}^{c} \leq y \leq \frac{b}{2}
\end{array} \quad\right. \text { if } & U_{c} \geq U_{c 2}^{c}
\end{array}\right.
$$

Finally, the rate of energy dissipated in the reinforcement crossing the full depth crack is given by:

$$
\dot{D}_{s c r}^{c}=\int_{0}^{b / 2} T_{s c r}^{c} \frac{\mathrm{d} \Delta_{s c r}^{c}}{\mathrm{~d} U_{c}} \mathrm{~d} y
$$

\subsubsection{Load deflection response}

The overall energy dissipated in the reinforcement, including bond-slip, across the various cracks is given by:

$$
\dot{D}_{s}^{c}=\dot{D}_{s c e}^{c}+\dot{D}_{s d y}^{c}+\dot{D}_{s d x}^{c}+\dot{D}_{s c r}^{c}
$$

where $\dot{D}_{s c e}^{c}, \dot{D}_{s d y}^{c}, \dot{D}_{s d x}^{c}$ and $\dot{D}_{s}^{c}$ are given by Equations (15), (25), (39) and (47) respectively.

It is assumed that the various parts of the slab bounded by the yield lines rotate rigidly. Under a uniformly distributed load $q$, the work done by the load is equal to $q$ multiplied by the volume created by the slab as it deforms. Thus, the rate of the work done by the external uniformly distributed load for the quarter of the slab is:

$$
\dot{E}=\frac{q a b(3-2 \eta)}{24}
$$


The present model employs a kinematic method of analysis [18] where the work done by the external forces $\dot{E}$ for an infinitesimal deflection should be equal to the energy dissipated within the slab $\dot{D}_{s}^{c}$, i.e.:

$$
\dot{E}=\dot{D}_{s}^{c}
$$

Using the above equation, the load-deflection response of an unrestrained slab can be readily established.

\subsubsection{Failure deflection}

Failure is defined as the point during loading when the stress in the reinforcement at the full depth crack at the centre of the slab reaches the ultimate strength of the steel. However, since the reinforcement stretches across both the full-depth crack and the diagonal yield line in the $x$ direction, the assessment of the deflection corresponding to failure is not straightforward. Thus two approaches are presented, with the first offering a more approximate yet simpler alternative.

\section{Failure approach 1: independent bond lengths}

Here it is assumed that as the slab deflects the bond lengths of the reinforcements spanning across the two cracks in the $x$-direction are not interacting. A conservative estimate of the failure can be obtained by assuming that the summation of the two crack widths, namely $\Delta_{\text {sdx }}^{c}$ and $\Delta_{s c r}^{c}$, in the $x$-direction corresponds to a single crack, and that the bond length for this crack can be fully developed over half the long span. This would correspond to the true behaviour in the case of a square slab and would be conservative for greater aspect ratios. The deflection corresponding to failure can be obtained by solving the following relationship for $U_{c}$ :

$$
\frac{\left(T_{u}-T_{y}\right)^{2}}{2 A_{s} E_{2} \sigma_{b}}=\Delta_{s d x}^{c}+\Delta_{s c r}^{c}
$$

Thus the failure deflection is given by: 


$$
\begin{aligned}
U_{c f 1 a}^{c} & =\frac{d \eta^{2} a^{2}}{d^{2}+\eta^{2} a^{2}}\left(1-\frac{\left(T_{u}-T_{y}\right)^{2}}{2 A_{s} E_{2} \sigma \eta a}\right) \times \\
& {\left[\sqrt{\left[1-\left(1+\frac{\eta^{2} a^{2}}{d^{2}}\right)\left(1-\frac{\eta^{2} a^{2}}{\left(\eta a-\frac{\left(T_{u}-T_{y}\right)^{2}}{2 A_{s} E_{2} \sigma}\right)^{2}}\right)\right.}\right] }
\end{aligned}
$$

However, for greater aspect ratios or higher bond strengths, this approach would result in unrealistically low failure deflections. For those cases, the assumptions used in the formulation of the reinforcement force in the $x$-direction would result in a more realistic failure deflection value. Thus, it is assumed in this case that failure occurs in the full depth crack, and that the bond length would have a limiting value defined by the line passing through the quarter point of the central yield line and the quarter point of the long span support (Figure 9). It can be easily shown that, except for the range of small deflections where the force in the reinforcement remains far less than the ultimate force, the reinforcement extension $\Delta_{s c r}^{c}$ along the full depth crack is more than the reinforcement extension $\Delta_{s d x}^{c}$ along the diagonal yield line. In addition to this, the bond length that is developed as a result of $\Delta_{s c r}^{c}$ at the full depth crack is limited only to one side of the crack, whereas for the diagonal yield line it can be developed under $\Delta_{s d x}^{c}$ to either side of the crack, thus resulting in smaller reinforcement forces even for equal values of $\Delta_{s d x}^{c}$ and $\Delta_{s c r}^{c}$. Thus, the failure is based on the reinforcement rupturing along the full depth crack. The deflection corresponding to this assumption can be obtained from the following condition:

$$
\left\{\begin{array}{llr}
\frac{\left(T_{u}-T_{y}\right)^{2}}{2 A_{s} E_{2} \sigma_{b}}=\Delta_{s c r}^{c} & \text { if } & \frac{T_{u}-T_{y}}{\sigma_{b}} \leq \frac{a}{4}(1-2 \eta) \\
\frac{\left(T_{u}-T_{y}\right) L}{A_{s} E_{2}}-\frac{\sigma_{b} L^{2}}{2 A_{s} E_{2}}=\Delta_{s c r}^{c} & \text { if } & \frac{T_{u}-T_{y}}{\sigma_{b}} \geq \frac{a}{4}(1-2 \eta)
\end{array}\right.
$$

Thus, the failure deflection using this alternative assumption is given as: 


$$
U_{c f 1 b}^{c}=\left\{\begin{array}{lll}
\sqrt{\frac{b^{2}}{4}-\left[\frac{b}{2}-\frac{\left(T_{u}-T_{y}\right)^{2} a}{2 A_{s} E_{2} \sigma_{b} b}\right]^{2}} & \text { if } & \frac{T_{u}-T_{y}}{\sigma_{b}} \leq \frac{a}{4}(1-2 \eta) \\
\sqrt{\frac{b^{2}}{4}-\left[\frac{b}{2}-\frac{a^{2}(1-2 \eta)}{4 A_{s} E_{2} b}\left(T_{u}-T_{y}-\frac{\sigma_{b} a}{8}(1-2 \eta)\right)\right]^{2}} & \text { if } & \frac{T_{u}-T_{y}}{\sigma_{b}} \geq \frac{a}{4}(1-2 \eta)
\end{array}\right.
$$

Finally, the conservative estimate would be chosen as the highest prediction from the two assumed alternatives:

$$
U_{c f 1}^{c}=\max \left\{U_{c f 1 a}^{c}, U_{c f 1 b}^{c}\right\}
$$

\section{Failure approach 2: interactive bond lengths}

In this approach, it is assumed that as the slab deflects the bond lengths meet at a point between the two crack, with the forces at the two crack faces affecting each other.

Initially, the cumulative bond lengths caused by the forces at the two cracks would be less than the available distance between the two cracks (Figure 10(a)). The force across the full depth crack at the centre of the slab and the force across the diagonal yield line in the $x$ direction are then respectively given by:

$$
\begin{aligned}
& T_{s d x \mid y=b / 2}^{c}=T_{y}+\sqrt{A_{s} E_{2} \sigma_{b} \Delta_{s d x \mid y=b / 2}^{c}} \\
& T_{s c r \mid y=b / 2}^{c}=T_{y}+\sqrt{2 A_{s} E_{2} \sigma_{b} \Delta_{s c r \mid y=b / 2}^{c}}
\end{aligned}
$$

The limit of this stage $U_{c l 1}^{c}$ is defined when the two bond lengths meet, which is given as the solution of the following equation for $U_{c}$ :

$$
\frac{T_{s c r \mid y=b / 2}^{c}-T_{y}}{\sigma_{b}}+\frac{T_{s d x \mid y=b / 2}^{c}-T_{y}}{\sigma_{b}}=\frac{a}{2}-\eta a
$$




$$
\Rightarrow U_{c l 1}^{c}=\sqrt{\Delta_{s d x \mid y=b / 2}^{c}}+\sqrt{2 \Delta_{s c \mid y=b / 2}^{c}}=\sqrt{\frac{\sigma_{b}}{A_{s} E_{2}}}\left(\frac{a}{2}-\eta a\right) \ldots
$$

Failure can occur at this stage either by rupture of the reinforcement across the full depth crack or across the diagonal yield line in the $x$-direction, although, as explained earlier, for typical slab configurations failure is expected to occur by rupture of the reinforcement along the full depth crack. Thus, the failure deflection is obtained by replacing $T_{u}$ for $T_{s d x \mid y=b / 2}^{c}$ and $T_{s c r \mid y=b / 2}^{c}$ in Equations (56) and (57) respectively, and solving for $U_{c}$ :

$$
U_{c f 2 a}=\min U_{c} \text { solution of }\left\{\begin{array}{l}
\frac{\left(T_{u}-T_{y}\right)^{2}}{A_{s} E_{2} \sigma_{b}}=\Delta_{s d x \mid y=b / 2}^{c} \\
\frac{\left(T_{u}-T_{y}\right)^{2}}{2 A_{s} E_{2} \sigma_{b}}=\Delta_{s c r \mid y=b / 2}^{c}
\end{array}\right.
$$

If failure does not occur before $U_{c l 1}^{c}$, after this point the bond lengths meet and change as the slab deflects. Considering the region between the two cracks, the reinforcement force drops from either side until a point where the two forces become equal and larger than the yield force of the reinforcement (Figure 10(b)). The distance from the diagonal yield line to this point is defined as $L_{1}$ and the remaining distance from the full depth crack defined as $L_{2}$. These conditions can be represented as:

$$
\begin{array}{ll}
L=L_{1}+L_{2} & \text { Condition } 1 \\
t_{1}-\sigma_{b} L_{1}=t_{2}-\sigma_{b} L_{2} & \text { Condition } 2
\end{array}
$$

where:

$$
\begin{aligned}
& L=\frac{a}{2}-\eta a \ldots \ldots \\
& t_{1}=T_{s d x \mid y=b / 2}^{c}-T_{y}
\end{aligned}
$$




$$
t_{2}=T_{s c r \mid y=b / 2}^{c}-T_{y}
$$

Moreover, the extension of the reinforcement at the diagonal yield line and the full depth crack can be obtained from:

$$
\Delta_{s}=\left\{\begin{array}{lll}
\frac{\left(T_{s}-T_{y}\right)^{2}}{2 A_{s} E_{2} \sigma_{b}} & \text { if } & l_{b} \leq L \\
\frac{\left(T_{s}-T_{y}\right) L}{A_{s} E_{2}}-\frac{\sigma_{b} L^{2}}{2 A_{s} E_{2}} & \text { if } & l_{b}=L
\end{array}\right.
$$

and can be formulated as:

$$
\begin{array}{ll}
t_{1}^{2}+2 \sigma_{b} L_{1} t_{1}-\sigma_{b}^{2} L_{1}^{2}=2 A_{s} E_{2} \sigma_{b} \Delta_{1} & \text { Condition } 3 \\
2 t_{2} L_{2}-\sigma_{b} L_{2}^{2}=2 A_{s} E_{2} \Delta_{2} & \text { Condition } 4
\end{array}
$$

where:

$$
\begin{aligned}
& \Delta_{1}=\Delta_{s d x \mid y=b / 2}^{c} \\
& \Delta_{2}=\Delta_{s c r \mid y=b / 2}^{c}
\end{aligned}
$$

This stage applies up to the point where the extension in the reinforcement across the diagonal yield line crack is only due to the slip to the left of the diagonal yield line without any contribution from the region between the cracks, that is $L_{1}=0$. By combining the Conditions presented above, the deflection $U_{c l 2}^{c}$ up to which this stage applies can be obtained by solving the following equation for $U_{c}$ :

$$
U_{c l 2}^{c}=U_{c} \text { solution of }\left(2 A_{s} E_{2} \Delta_{2}-\sigma_{b} L^{2}=\sqrt{8 A_{s} E_{2} \sigma_{b} \Delta_{1}} L\right) .
$$

Failure occurs during this stage by rupture of the reinforcement across either of the two 
cracks. Consider:

$$
t_{u}=T_{u}-T_{y}
$$

then assuming that failure occurs by rupture across the diagonal yield line, the deflection corresponding to this case would be obtained by substituting $t_{u}$ for $t_{1}$ in the aforementioned Conditions and solving these simultaneously for the unknown parameters $\left(t_{2}, L_{1}, L_{2}, U_{c}\right)$. However, due to the nonlinear nature of the Conditions, the solution can only be obtained using an iterative method. Similarly, assuming that failure occurs by rupture of the reinforcement across the full depth crack, the deflection corresponding to this rupture is obtaining by substituting $t_{u}$ for $t_{2}$ and solving the four Conditions simultaneously for the unknown parameters $\left(t_{1}, L_{1}, L_{2}, U_{c}\right)$. Accordingly:

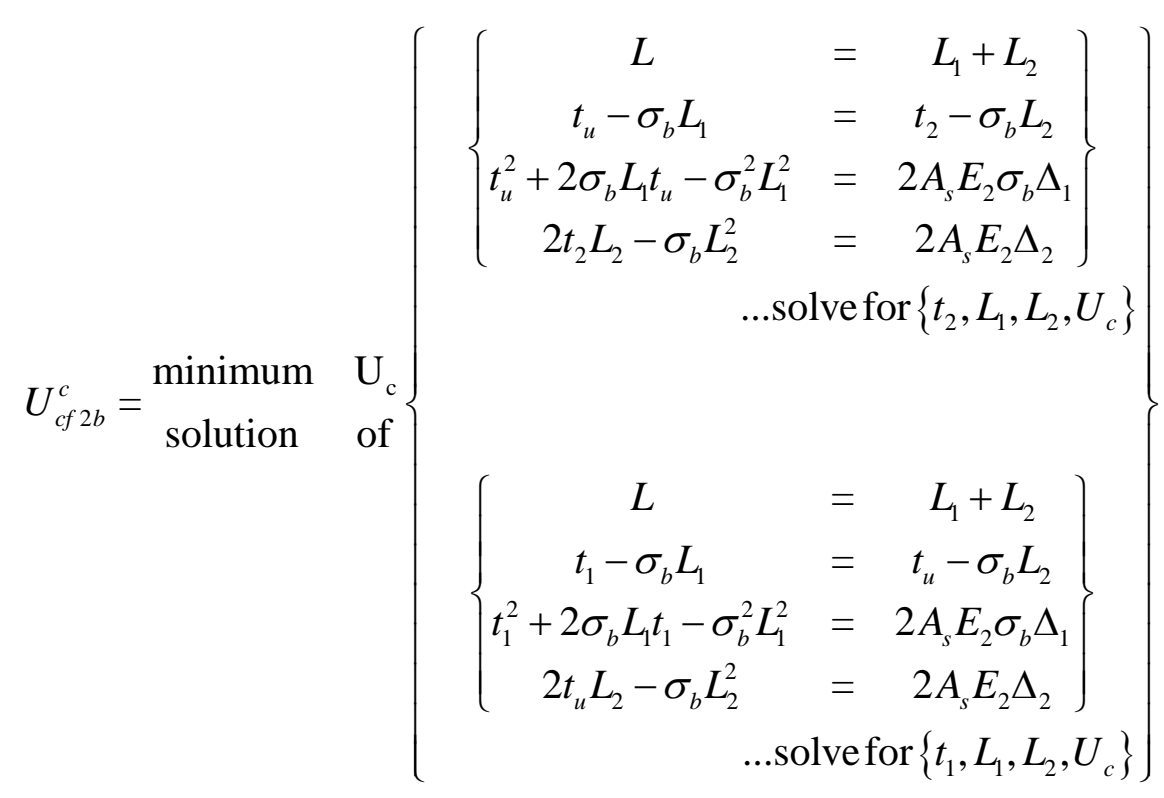

If failure does not occur during stage 2, then as the slab deflects further the reinforcement force drops monotonically from the full depth crack to the intersection of the diagonal cracks and continues to drop over the triangular part of the slab (Figure 10(c)). This effectively means that the extension of the reinforcement at the full depth crack is large enough to require slip in both the triangular and trapezoidal regions to occur in the same direction from the edge towards the 
centre of the slab. Thus, the two cracks can be treated as a single crack and the deflection $U_{c f 2 c}^{c}$ corresponding to failure can be obtained as:

$$
\begin{aligned}
U_{c f 2 c}^{c} & =\frac{d \eta^{2} a^{2}}{d^{2}+\eta^{2} a^{2}}\left(1-\frac{\left(T_{u}-T_{y}\right)^{2}}{2 A_{s} E_{2} \sigma \eta a}\right) \times \\
& {\left[1+\sqrt{1-\left(1+\frac{\eta^{2} a^{2}}{d^{2}}\right)\left(1-\frac{\eta^{2} a^{2}}{\left(\eta a-\frac{\left(T_{u}-T_{y}\right)^{2}}{2 A_{s} E_{2} \sigma}\right)^{2}}\right)}\right] }
\end{aligned}
$$

Therefore, considering the three stages of response, the failure deflection $U_{c f}^{c}$ for the CM model is given for the second alternative approach by:

$$
U_{c f 2}^{c}=\left\{\begin{array}{lll}
U_{c f 2 a}^{c} & \text { if } & U_{c f 2 a}^{c} \leq U_{c l 1}^{c} \\
U_{c f 2 b}^{c} & \text { if } & U_{c l 1}^{c} \leq U_{c f 2 b}^{c} \leq U_{c l 2}^{c} \\
U_{c f 2 c}^{c} & \text { if } & U_{c l 2}^{c} \leq U_{c f 2 c}^{c}
\end{array}\right.
$$

\subsection{Intersection Crack Model}

For the IM model, assumptions similar to the CM model are employed. The model is based on the assumption that as the slab deflects under loading, if a full depth crack opens running in the short span direction at the intersection of the yield lines, the assumed rigid parts of the slab would comprise of triangular and rectangular parts (Figure 2(b)). The triangular parts are in contact at the corner of the slab at the top fibre, and they rotate by pushing the slab out and below the top surface, as previously depicted in Figure 7. For the complete formulation of the IM model, the reader is referred to [17]. Hereafter, simplified forms are presented for both the CM and IM models. 


\section{Simplified Ambient Temperature Models}

The proposed CM and IM models can be too cumbersome in their full form for designoriented application, since they involve integration and rather complex logic for the selection of appropriate expressions. Here, simplifications are proposed for the two models, similar to ones presented for the restrained slab model [10], which aim at providing a practical design-oriented tool for failure assessment of unrestrained LRC slabs.

As in the restrained slab model [10], it is assumed that a strip across the centre of each region is a representative average of the behaviour along that region. Moreover, the bond lengths of each strip are assumed to be unbounded, and the reinforcement is assumed to be sufficiently long so that the stress drops to the yield strength of the steel. Accordingly, using an approximation of the type given by Equation (35), the extension in the reinforcement at the chosen point is given by the following expressions for the CM model:

$$
\begin{aligned}
& \Delta_{s c e}^{c}=\frac{2 d U_{c}}{b}+\frac{U_{c}^{2}(1-2 \eta)}{2 b} \\
& \Delta_{s d y}^{c}=\frac{2 d U_{c}}{b}+\frac{U_{c}^{2}(1-2 \eta)}{2 b} \\
& \Delta_{s d x}^{c}=\frac{d U_{c}}{\eta a}+\frac{U_{c}^{2}(1-2 \eta)}{4 \eta a} \\
& \Delta_{s c r}^{c}=\frac{U_{c}^{2}}{2 a}
\end{aligned}
$$

Similarly, the relevant expressions for reinforcement extension in the IM model are obtained as:

$$
\begin{aligned}
\Delta_{s c e}^{i} & =\frac{2 d U_{c}}{b} \\
\Delta_{s d y}^{i} & =\frac{2 d U_{c}}{b}
\end{aligned}
$$




$$
\begin{aligned}
\Delta_{\text {sdx }}^{i} & =\frac{d U_{c}}{\eta a} \\
\Delta_{s c r}^{i} & =\frac{U_{c}^{2}}{4 \eta a}
\end{aligned}
$$

Moreover, the respective reinforcement forces are obtained for the CM model as follows:

$$
\begin{aligned}
& T_{s c e}^{c}=T_{y}+\sqrt{2 A_{s} E_{2} \sigma_{b} \Delta_{s c e}^{c}} \\
& T_{s y}^{c}=T_{y}+\sqrt{A_{s} E_{2} \sigma_{b} \Delta_{s d y}^{c}} \cdots \\
& T_{s x}^{c}=T_{y}+\sqrt{A_{s} E_{2} \sigma_{b} \Delta_{s d x}^{c}} \cdots \\
& T_{s c r}^{c}=T_{y}+\sqrt{2 A_{s} E_{2} \sigma_{b} \Delta_{s c r}^{c}}
\end{aligned}
$$

and for the IM model as follows:

$$
\begin{aligned}
& T_{s c e}^{i}=T_{y}+\sqrt{2 A_{s} E_{2} \sigma_{b} \Delta_{s c e}^{i}} \\
& T_{s y}^{i}=T_{y}+\sqrt{A_{s} E_{2} \sigma_{b} \Delta_{s d y}^{i}} \cdots \\
& T_{s x}^{i}=T_{y}+\sqrt{A_{s} E_{2} \sigma_{b} \Delta_{s d x}^{i}} \cdots \\
& T_{s c r}^{i}=T_{y}+\sqrt{A_{s} E_{2} \sigma_{b} \Delta_{s c r}^{i}} \ldots
\end{aligned}
$$

Therefore, the load deflection response is described by the following simplified relationships for the CM and the IM models, respectively:

$$
q^{c}=12 \frac{\left[T_{s c e}^{c}(1-2 \eta) \alpha+2 T_{s y}^{c} \eta \alpha+\frac{T_{s x}^{c}}{2 \eta \alpha}\right]\left[2 d+(1-2 \eta) U_{c}\right]+T_{s c r}^{c} \frac{U_{c}}{\alpha}}{a b(3-2 \eta)}
$$




$$
q^{i}=24 \frac{T_{s c e}^{i}(1-2 \eta) \alpha d+2 T_{s y}^{i} \eta \alpha d+\frac{T_{s x}^{i} d}{2 \eta \alpha}+T_{s c r}^{i} \frac{U_{c}}{4 \eta \alpha}}{a b(3-2 \eta)}
$$

For the CM model, the failure deflection is obtained by simplifying the expression developed in Section 2.1.6 using Equation (35), leading to:

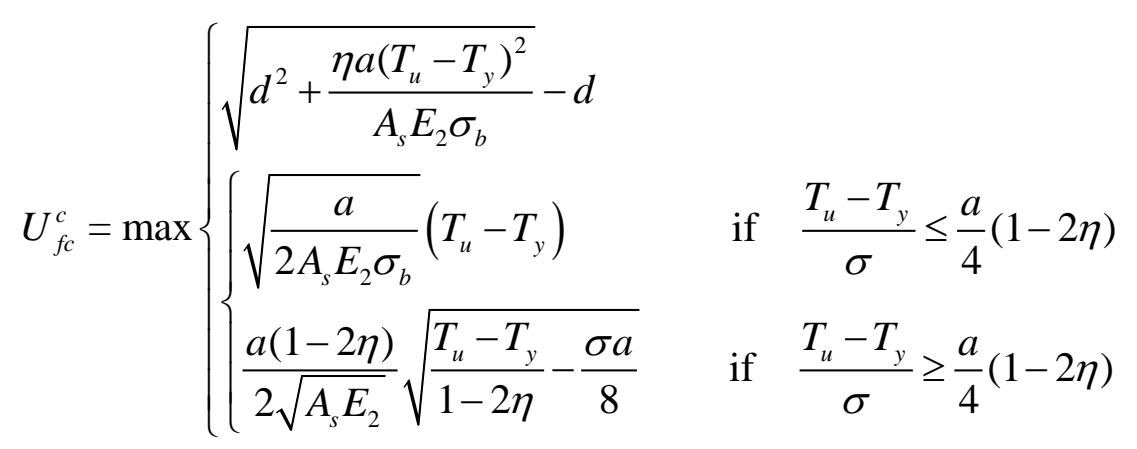

For the IM model, it is assumed that the slab fails by rupture of the reinforcement at the intersection of the yield lines stretching in the long direction. At this location, the full-depth crack meets the diagonal yield line crack and thus the total reinforcement extension is given by the summation of these two cracks. The deflection corresponding to failure can be obtained from the following equation of reinforcement force where the first corresponds to the case of unbounded bond length and the second to the case of the bond length being bounded on one side and unbounded on the other:

$$
\begin{aligned}
& T_{u}=T_{y}+\sqrt{A_{s} E_{2} \sigma_{b}\left(\Delta_{s d x}^{i}+\Delta_{s c r}^{i}\right)} \\
& T_{u}=T_{y}-\sigma_{b}\left(\frac{a}{2}-\eta a\right)+\sqrt{2 \sigma_{b}^{2}\left(\frac{a}{2}-\eta a\right)^{2}+2 A_{s} E_{2} \sigma_{b}\left(\Delta_{s d x}^{i}+\Delta_{s c r}^{i}\right)}
\end{aligned}
$$

Thus, the failure deflection is obtained as:

$$
U_{c f}^{i}=\frac{d \eta^{2} a^{2}(1-\Gamma)}{d^{2}+\eta^{2} a^{2}}\left[\sqrt{1-\left(1+\frac{\eta^{2} a^{2}}{d^{2}}\right)\left(1-\frac{1}{(1-\Gamma)^{2}}\right)}-1\right]
$$


where

$$
\Gamma=\left\{\begin{array}{lll}
\frac{\left(T_{u}-T_{y}\right)^{2}}{A_{s} E_{2} \sigma_{b} \eta a} & \text { if } & \frac{T_{u}-T_{y}}{\sigma_{b}} \leq \frac{a}{2}-\eta a \\
\frac{\left[2\left(T_{u}-T_{y}\right)+\sigma_{b} a(1-2 \eta)\right]^{2}-2 \sigma_{b}^{2} a^{2}(1-2 \eta)^{2}}{8 A_{s} E_{2} \sigma_{b} \eta a} & \text { if } & \frac{T_{u}-T_{y}}{\sigma_{b}} \geq \frac{a}{2}-\eta a
\end{array}\right.
$$

The failure deflection for the IM model is obtained by simplifying Equation (95) as:

$$
U_{c f}^{i}=\sqrt{d^{2}+\frac{\eta a(1-2 \eta)^{2}}{4 A_{s} E_{2} \sigma_{b}} \Gamma^{i a}}-d
$$

where,

$$
\Gamma^{i a}=\left\{\begin{array}{lll}
8\left(\frac{T_{u}-T_{y}}{1-2 \eta}\right)^{2} & \text { if } & \frac{T_{u}-T_{y}}{\sigma_{b}} \leq \frac{a}{2}-\eta a \\
\left(\frac{2\left(T_{u}-T_{y}\right)}{1-2 \eta}+\sigma_{b} a\right)^{2}-2 \sigma_{b}^{2} a^{2} & \text { if } & \frac{T_{u}-T_{y}}{\sigma_{b}} \geq \frac{a}{2}-\eta a
\end{array}\right.
$$

\section{Simplified elevated temperature model}

The assessment of the response of a LRC slab under elevated temperature requires the modelling of the thermal expansion and thermal curvature of the concrete along with the deterioration of the material properties. In developing the model for elevated temperature, the load-deflection response at a given temperature distribution is sought assuming uniform heating along the extent of the slab. The more realistic scenario where the temperature is varied at constant loading can be inferred from the family of load-deflection curves corresponding to given temperatures.

The slab here is again treated as consisting of a series of beam strips. As the slab is 
loaded, a crack forms across a strip with increasing deflections similar to the ambient case. However, this crack will not form immediately since the LRC slab expands. After a certain deflection, the central crack penetrates the depth of the strip, and the two halves of the strip will be curved separately. Here, for simplicity, it is assumed that the temperature gradient is linear and that the temperature of the reinforcement and the surrounding concrete is the same at the level of the reinforcement, similar to the assumption made by [11]. Should it be deemed necessary, a different temperature distribution could be easily employed in subsequent extensions of this work; however, the simplified linear temperature distribution is considered here to offer a good balance between accuracy and model simplicity. The extent of the curvature of the strip is determined by the thermal properties of the concrete. In addition to this, it is assumed that each half of the strip forms a separate curve throughout the loading. This can result in an error in the initial stages of the loading involving bending action. However, in the tensile catenary stage when a full depth crack is formed, and where failure is expected to occur this assumption becomes realistic.

Figure 11 shows the thermally curved configuration of the strip. Due to the increased temperature the strip will expand. In addition to this, the strip will curve and try to pull-in due to the temperature gradient. The expansion of the strip at the level of the reinforcement $\Delta l_{e}$ is given by:

$$
\Delta l_{e}=\alpha_{c} \Delta T_{r} L
$$

where $\alpha_{c}$ is the coefficient of thermal expansion of the concrete and $\Delta T_{r}$ is the temperature at the level of the reinforcement. The pull-in of the strip at the level of the reinforcement $\Delta l_{p}$ is given by [11] as:

$$
\begin{aligned}
& \Delta l_{p}=\int_{0}^{L} \frac{1}{2}\left(\frac{\mathrm{d} y}{\mathrm{~d} x}\right)^{2} d x \\
& \Rightarrow \Delta l_{p}=\frac{\kappa^{2} L^{3}}{24} \ldots \ldots \ldots . . .
\end{aligned}
$$


where $\kappa$ is the the thermal curvature of the the strip assuming linear temperature distribution across the depth and is obtained as:

$$
\kappa=\frac{\Delta T_{t b} \alpha_{c}}{h}
$$

in which $\Delta T_{t b}$ is the temperature difference between the top and the bottom of the strip and $h$ is the height of the beam section. Therefore, the length of the chord $L_{c}$ joining the two ends of the strip is:

$$
L_{c}=L+\Delta l_{e}-\Delta l_{p}
$$

Referring to Figure 12, the extension of the reinforcement at elevated temperatures can thus be obtained as:

$$
\Delta_{s}=L-\sqrt{L_{c}^{2}-U^{2}}
$$

In extending the unrestrained slab model to deal with the fire condition, the effect of elevated temperatures on the material properties and on the thermally curved geometry of the LRC slab are considered. The increase in temperature causes the concrete slab to expand and curve in addition to causing differential expansion between the reinforcement and the surrounding concrete due to different coefficients of thermal expansion for steel and concrete. The following assumptions are made for the elevated temperature model:

- the failure mechanism is unchanged from the ambient case, and is defined by yield lines determined according to yield line theory,

- the temperature distribution is uniform over the slab length and width, but varies linearly over the slab depth,

- the parts of the slabs bounded by the yield lines are rigid, but the underlying shape can be curved and expanded from the ambient case due to thermal curvature/expansion effects, governed by the thermal expansion characteristics of concrete. 
The elevated temperature model is formulated expressing the load-deflection response and the failure expression at a specific level of temperature. This can be readily used to establish the temperature-deflection response and the failure temperature for specific loading.

It is assumed that the slab is free to expand in any direction since it is unrestrained, that it is free to curve in the direction perpendicular to the slab edges, and that strips parallel to the slab edges can curve freely at the interior end of the slab part decreasing to no curvature at the slab supports (Figure 13). The extension of the CM and IM models, developed in the previous section for the ambient temperature unrestrained slab, is presented hereafter for elevated temperature.

Simplified versions of the proposed elevated temperature models for unrestrained slabs are presented, following similar lines to those adopted for the ambient models. Accordingly, it is assumed that strips taken across the mid-point of every region represent the behaviour over that region, thus providing considerably simplified formulations. The reader is referred to [17] for the detailed versions of the elevated temperature CM and IM models.

\subsection{CM model}

Applying the simplification of Equation (35), the planar rotation of the slab is obtained as:

$$
\phi_{c}=\frac{2 U_{c}^{2}}{a b}+\frac{\kappa b}{a}\left(d+\frac{\kappa b^{2}}{96}\right) .
$$

The extension of the reinforcement at the centre of the four considered regions is given by:

$$
\begin{aligned}
& \Delta_{s c e}^{c}=d\left(\frac{2 U_{c}}{b}-\frac{\kappa b}{4} \sqrt{1-\frac{4 U_{c}^{2}}{b^{2}}}\right)+\phi_{c} \frac{a}{4}(1-2 \eta) \ldots \ldots \ldots \ldots . . . . . . . . \\
& \Delta_{s d y}^{c}=\frac{U_{c}^{2}}{2 b}+d\left(\frac{2 U_{c}}{b}+\frac{\kappa b}{4} \sqrt{1-\frac{4 U_{c}^{2}}{b^{2}}}\right)+\frac{3 \kappa^{2} b^{3}}{1536}-\phi_{c} \frac{\eta a}{2}
\end{aligned}
$$




$$
\begin{aligned}
& \Delta_{s d x}^{c}=\frac{U_{c}^{2}}{4 \eta a}+d\left(\frac{U_{c}}{\eta a}+\frac{\kappa \eta a}{2} \sqrt{1-\frac{U_{c}^{2}}{\eta^{2} a^{2}}}\right)+\frac{\kappa^{2} a^{3}}{1536}\left[8 \eta^{3}+(1-\eta)^{3}\right]-\phi_{c} \frac{b}{4} \\
& \Delta_{s c r}^{c}=\phi_{c} \frac{b}{4}+\frac{\kappa^{2} a^{3}}{1536}(1-\eta)^{3}
\end{aligned}
$$

Thus, assuming that the reinforcement expands differentially along half the length and the width of the slab, the reinforcement force is obtained as:

$$
\begin{aligned}
& T_{s c e}^{c}=T_{y}+\sqrt{2 A_{s} E_{2} \sigma_{b}\left[\Delta_{s c e}^{c}+\varepsilon_{s c t} \frac{b}{4}\right]} \\
& T_{s y}^{c}=T_{y}+\sqrt{A_{s} E_{2} \sigma_{b}\left[\Delta_{s d y}^{c}+\varepsilon_{s c t} \frac{b}{4}\right]} . \\
& T_{s x}^{c}=T_{y}+\sqrt{A_{s} E_{2} \sigma_{b}\left(\Delta_{s d x}^{c}+\varepsilon_{s c t} \frac{a}{4}\right)} \\
& T_{s c r}^{c}=T_{y}+\sqrt{2 A_{s} E_{2} \sigma_{b}\left(\Delta_{s c r}^{c}+\varepsilon_{s c t} \frac{a}{4}(1-\eta)\right)}
\end{aligned}
$$

in which:

$$
\varepsilon_{s c t}=\left(\alpha_{c}-\alpha_{s}\right) \Delta T_{r}
$$

where $\alpha_{s}$ is the coefficient of thermal expansion of steel reinforcement.

Therefore, the load deflection response is given by:

$$
q^{c}=24 \frac{T_{s c e} \frac{\mathrm{d} \Delta_{s c e}}{\mathrm{~d} U_{c}} \frac{a}{2}(1-2 \eta)+T_{s y} \frac{\mathrm{d} \Delta_{s d y}}{\mathrm{~d} U_{c}} \eta a+\left(T_{s x} \frac{\mathrm{d} \Delta_{s d x}}{\mathrm{~d} U_{c}}+T_{s c r} \frac{\mathrm{d} \Delta_{s c r}}{\mathrm{~d} U_{c}}\right) \frac{b}{2}}{a b(3-2 \eta)} .
$$

and the failure deflection is simplified to: 


$$
U_{c f}^{c}=\max \left\{\begin{array}{l}
\sqrt{\frac{\eta a\left(T_{u}-T_{y}\right)^{2}}{A_{s} E_{2} \sigma_{b}}-\varepsilon_{s c t} \frac{\eta a^{2}}{2}+d^{2}-\frac{\kappa^{2} \eta a^{4}}{96}\left[(1-2 \eta)^{2}+8 \eta^{3}\right]}-d \\
\sqrt{\Gamma^{c e}-\varepsilon_{s c t} \frac{a^{2}}{4}(1-2 \eta)-\frac{\kappa^{2} a^{4}}{384}-\frac{\kappa b^{2}}{2}\left(d+\frac{\kappa b^{2}}{96}\right)}
\end{array}\right.
$$

where:

$$
\Gamma^{c e}=\left\{\begin{array}{lll}
\frac{\left(T_{u}-T_{y}\right)^{2} a}{2 A_{s} E_{2} \sigma_{b}} & \text { if } & \frac{T_{u}-T_{y}}{\sigma} \leq \frac{a}{4}(1-2 \eta) \\
\frac{\left(T_{u}-T_{y}\right) a^{2}(1-2 \eta)}{4 A_{s} E_{2}}-\frac{\sigma_{b} a^{3}(1-2 \eta)^{2}}{32 A_{s} E_{2}} & \text { if } & \frac{T_{u}-T_{y}}{\sigma} \geq \frac{a}{4}(1-2 \eta)
\end{array}\right.
$$

\subsection{IM model}

For the IM model, the planar rotation is simplified to:

$$
\phi_{i}=\frac{U_{c}^{2}}{\eta a b}+\frac{\kappa b}{2 \eta a}\left(d+\frac{\kappa b^{2}}{96}\right)
$$

whereas the reinforcement extension in the four considered regions is obtained as:

$$
\begin{aligned}
& \Delta_{\text {sce }}^{i}=d\left(\frac{2 U_{c}}{b}-\frac{\kappa b}{4} \sqrt{1-\frac{4 U_{c}^{2}}{b^{2}}}\right) \\
& \Delta_{s d y}^{i}=\frac{U_{c}^{2}}{2 b}+d\left(\frac{2 U_{c}}{b}+\frac{\kappa b}{4} \sqrt{1-\frac{4 U_{c}^{2}}{b^{2}}}\right)+\frac{3 \kappa^{2} b^{3}}{1536}-\phi_{i} \frac{\eta a}{2} \\
& \Delta_{\text {sdx }}^{i}=\frac{U_{c}^{2}}{4 \eta a}+d\left(\frac{U_{c}}{\eta a}+\frac{\kappa \eta a}{2} \sqrt{1-\frac{U_{c}^{2}}{\eta^{2} a^{2}}}\right)+\frac{3 \kappa^{2} \eta^{3} a^{3}}{512}-\phi_{i} \frac{b}{4} \\
& \Delta_{s c r}^{i}=\phi_{i} \frac{b}{4}+\frac{\kappa^{2} a^{3}}{1536}\left(\eta^{3}+8(1-2 \eta)^{3}\right)
\end{aligned}
$$


Assuming that for the differential expansion the midpoint between the diagonal yield line crack and the full depth crack along the short direction is considered as the anchorage point for the reinforcement, the reinforcement force is expressed as:

$$
\begin{aligned}
& T_{s c e}^{i}=T_{y}+\sqrt{2 A_{s} E_{2} \sigma_{b}\left[\Delta_{s c e}^{i}+\varepsilon_{s c t} \frac{b}{4}\right]} \\
& T_{s y}^{i}=T_{y}+\sqrt{A_{s} E_{2} \sigma_{b}\left[\Delta_{s d y}^{i}+\varepsilon_{s c t} \frac{b}{4}\right]} \text {. } \\
& T_{s x}^{i}=T_{y}+\sqrt{A_{s} E_{2} \sigma_{b}\left[\Delta_{s d x}^{i}+\varepsilon_{s c t} \frac{\eta a}{2}\right]} \\
& T_{s c r}^{i}=T_{y}+\sqrt{A_{s} E_{2} \sigma_{b}\left(\Delta_{s c r}^{i}+\varepsilon_{s c t} \frac{a(1-\eta)}{4}\right)}
\end{aligned}
$$

Accordingly, the load deflection response is obtained from:

$$
q^{i}=24 \frac{T_{s c e} \frac{\mathrm{d} \Delta_{s c e}}{\mathrm{~d} U_{c}} \frac{a}{2}(1-2 \eta)+T_{s y} \frac{\mathrm{d} \Delta_{s d y}}{\mathrm{~d} U_{c}} \eta a+\left(T_{s x} \frac{\mathrm{d} \Delta_{s d x}}{\mathrm{~d} U_{c}}+T_{s c r} \frac{\mathrm{d} \Delta_{s c r}}{\mathrm{~d} U_{c}}\right) \frac{b}{2}}{a b(3-2 \eta)}
$$

whereas the failure deflection is obtained as:

$$
U_{c f}^{i}=\sqrt{d^{2}-\varepsilon_{s c t} \frac{\eta a^{2}}{2}-\frac{\kappa^{2} \eta a^{4}}{24}\left[2 \eta^{3}+(1-2 \eta)^{3}\right]+2 \eta a \Gamma^{i e}}-d
$$

in which: 


$$
\Gamma^{i e}=\left\{\begin{array}{lll}
\frac{\left(T_{u}-T_{y}\right)^{2}}{A_{s} E_{2} \sigma_{b}} & \text { if } & \frac{T_{u}-T_{y}}{\sigma_{b}} \leq \frac{a}{2}(1-2 \eta) \\
\frac{\left(T_{u}-T_{y}\right)^{2}}{2 A_{s} E_{2} \sigma_{b}}+\frac{\left(T_{u}-T_{y}\right) a(1-2 \eta)}{2 A_{s} E_{2}}-\frac{\sigma_{b} a^{2}}{8 A_{s} E_{2}}(1-2 \eta)^{2} & \text { if } & \frac{T_{u}-T_{y}}{\sigma_{b}} \geq \frac{a}{2}(1-2 \eta)
\end{array}\right.
$$

\section{Comparative Examples}

The various proposed models are compared hereafter to the results of detailed nonlinear finite element analysis using ADAPTIC [19] as well as to the predictions of the BRE simplified slab model [16] where possible. The companion paper [12] presents detailed verification and validation of the proposed models, discusses their application, and highlights any restrictions in relation to their applicability.

\subsection{Detailed ambient temperature models}

Conisderation is given here to the ambient temperature response of a simply supported unrestrained slab. The material model chosen is stl4 for steel and con11 for concrete [20], along with the cover variant of the concrete slab element csl4 [20],[21]. Two sets of comparisons are

presented, where only the long span is different with all other parameters kept constant. The material properties are given in

Table 1 and Table 2, while the geometric properties are as given in Table 3.

Due to symmetry only one quarter of the slab is modeled with ADAPTIC . The slab is simply supported along the edges and free to move in-plane. Moreover, appropriate boundary conditions are applied along the axes of symmetry. Square csl4 slab elements are used with a side length of $375 \mathrm{~mm}$ each. Figure 14 depicts the ADAPTIC model for $a=12 \mathrm{~m}$.

The reinforcement chosen corresponds to bars with a diameter of $12 \mathrm{~mm}$ spaced at $250 \mathrm{~mm}$. According to [22], this corresponds to a reinforcement perimetric design value of bond strength of $1.8 \mathrm{MPa}$ or equivalently $0.27 \mathrm{~N} / \mathrm{mm}$ per mm width of the slab. The load-deflection response obtained with ADAPTIC is compared against the proposed models in Figures 15 and 
16, for $a=9 m$ and $a=12 m$ respectively, where 'high bond' and 'low bond' refer to the $0.45 \mathrm{~N} / \mathrm{mm}$ and $0.15 \mathrm{~N} / \mathrm{mm}$, respectively, per mm width of the slab. It can be observed in every case that the CM model predicts a stiffer response compared to the IM model, and that the IM model predicts a stiffer response compared to the BRE model [16]. This can be attributed to the fact that the current formulations allow for an increase in the reinforcement force, while the BRE method assumes constant force corresponding to the yield strength of the steel with a constant increase of $10 \%$ along the through depth crack. Moreover, it can be observed that the current models compare better with the nonlinear finite element results than the BRE model.

The failure deflection and the corresponding loads obtained using the various approaches are summarized in Table 4 for $a=9 m$ and Table 5 for $a=12 m$. For the CM model, consideration is given to the two failure prediction approaches presented in Section 2.1.6. It can be observed for both the CM and IM models that higher bond strength leads to a reduction in the failure deflection as expected, while the BRE model employs an empirical estimate which does not account for bond. Moreover, for a given slab geometry and bond strength, the failure displacements obtained from the proposed models do not differ significantly, indicating that the long span does not affect the failure displacement. On the other hand, the failure displacement predicted by the BRE model are relatively small and can only be realistic for relatively large bond strength.

\subsection{Simplified ambient temperature models}

For the simplified models applied to the slab described previously with $a=9 m$ and $\sigma_{b}=0.15 \mathrm{~N} / \mathrm{mm}^{2}$, the load-deflection response predictions from the full and simplified model formulations are depicted in Figure 17, while the failure deflections are compared in Table 6.

It can be observed that the simplified model variants compare very well against the full formulations. The response up to a deflection of around $600 \mathrm{~mm}$ is almost identical, deviating only slightly afterwards. Thus, it is proposed that the simplified model variants are used for practical design applications, as they utilise significantly simpler expressions while maintaining a good level of accuracy in comparison with the full model variants.

\subsection{Simplified elevated temperature models}


Considering the elevated temperature response of the slab described previously with $a=9 \mathrm{~m}$, the parameters given in Table 7 and a bond strength of $0.05 \mathrm{~N} / \mathrm{mm}^{2}$, the load deflection responses obtained using the full and the simplified formulation are depicted in Figure 18. The bond strength value selected here is lower than the value used previously for the ambient temperature cases reflecting the fact that material properties deteriorate at elevated temperatures. Since there is a lack of largely accepted data related to bond strength variation with temperature this choice is made consistently with the reduction in concrete strength at elevated temperature.

It can be observed that, similar to the previous models, the proposed simplified versions provide very good comparisons against the full models, deviating only slightly after relatively large deflections, in this case at around $700 \mathrm{~mm}$ of central deflection. Moreover, the failure deflections predicted by the full and the simplified version are very close, as shown in Table 8, thus demonstrating the reliability of the simplified models under elevated temperature.

\section{Conclusions}

This paper presents model aimed at capturing the behaviour of unrestrained LRC floor slabs under ambient temperature. Two variants of the model are developed based on different failure modes. Ambient temperature model varinats are initially developed, and these are subsequently extended to account for the effects of elevated temperature.

The proposed models account for membrane action in simply supported unrestrained slabs through considering the effects of changing geometry at large deflections. Importantly, the effect of the bond strength on the response is included, which for LRC members is one of the parameters governing the rupture of the reinforcement and hence the failure of the whole slab. The bond stress developed between the reinforcement and the surrounding concrete affects the level of strain concentration in the reinforcing bars, an effect which has thus far been ignored by design codes and recently developed design models for floor slabs under fire.

The paper provides simplified formulations of the model variants, which are shown to provide favourable comparisons against the full formulations as well as detailed non-linear finite element analyses. The post-cracking response at large deflections is obtained, and a ductility limit on the response is established which reflects the rupture of the reinforcement across the 
cracks developed at large deflections.

The ability of the proposed models to capture the large deflection response of simply supported unrestrained slabs and the definition of a failure criterion based on reinforcement rupture is an important step towards the rational performance-based design of composite floor slabs under elevated temperature. An important outcome of the application of the proposed models is the identification of bond strength as an important paramater governing reinforcement rupture, thus highlighting the need for further research into bond characteristics particularly under elevated temperatures.

The companion paper elaborates on the failure mechanisms assumed in this paper, where the kinematic assumptions underlying the proposed models are verified. Further comparisons against previously developed models as well as test results are also presented, and the applicability of the various models is examined and demonstrated. 


\section{Tables}

Table 1 Material properties for concrete (con11) at ambient temperatures [11],[20]

\begin{tabular}{|l|c|}
\hline Elastic modulus & $30 \mathrm{GPa}$ \\
\hline Poisson's ratio & 0.2 \\
\hline Compressive strength & $40 \mathrm{MPa}$ \\
\hline Tensile softening modulus & $300 \mathrm{MPa}$ \\
\hline Tensile strength, $f_{t 1}$ & $0.2 \mathrm{MPa}$ \\
\hline Tensile strength, $f_{t 2}$ & $2.0 \mathrm{MPa}$ \\
\hline Initial compressive nonlinearity parameter & 0.4 \\
\hline Residual postcrushing strength parameter & 0.2 \\
\hline Compressive interaction parameter & 0.6 \\
\hline Shear retention factor & 0.5 \\
\hline Shear interaction parameter & 0.4 \\
\hline Shear softening parameter & 0.0 \\
\hline
\end{tabular}

Table 2 Material properties for steel (stl4) at ambient temperatures

\begin{tabular}{|c|c|c|c|}
\hline $\mathrm{T}_{y}$ & $\mathrm{~T}_{u}$ & $\mathrm{E}_{2}$ & Rupture strain \\
\hline $180 \mathrm{~N} / \mathrm{mm}$ & $270 \mathrm{MPa}$ & $1050 \mathrm{MPa}$ & 0.19 \\
\hline
\end{tabular}

Table 3 Geometric properties for the unrestrained slabs

\begin{tabular}{|c|c|c|c|}
\hline$a$ & $b$ & $d$ & $A_{s}$ \\
\hline $6 \mathrm{~m}, 12 \mathrm{~m}$ & $6 \mathrm{~m}$ & $120 \mathrm{~mm}$ & $0.45 \mathrm{~mm}^{2} / \mathrm{mm}$ \\
\hline
\end{tabular}


Table 4 Failure deflection $(\mathrm{mm})$ and load $\left(\mathrm{kN} / \mathrm{m}^{2}\right)$ for the slab with $a=9 \mathrm{~m}$ under ambient temperature

\begin{tabular}{|c|c|c|c|c|c|c|c|}
\hline$\sigma_{b}$ & \multicolumn{3}{|c|}{ CM } & \multicolumn{3}{c|}{ IM } & \multicolumn{2}{c|}{ BRE } \\
\hline \multirow{2}{*}{0.15} & approach & $U_{f c}$ & $q$ & $U_{f c}$ & $q$ & $U_{f c}$ & $q$ \\
\cline { 2 - 7 } & 1 & 694 & 33.08 & 787 & 30.13 & 170 & 12.07 \\
\hline 0.45 & 1 & 711 & 33.76 & & & & \\
\cline { 2 - 5 } & 2 & 413 & 26.02 & 414 & 22.03 & & \\
\hline
\end{tabular}

Table 5 Failure deflection $(\mathrm{mm})$ and $\operatorname{load}\left(\mathrm{kN} / \mathrm{m}^{2}\right)$ for the slab with $a=12 \mathrm{~m}$ under ambient temperature

\begin{tabular}{|c|c|c|c|c|c|c|c|}
\hline$\sigma_{b}$ & \multicolumn{3}{|c|}{ CM } & \multicolumn{2}{c|}{ IM } & \multicolumn{2}{c|}{ BRE } \\
\hline \multirow{2}{*}{0.15} & approach & $U_{f c}$ & $q$ & $U_{f c}$ & $q$ & $U_{f c}$ & $q$ \\
\cline { 2 - 7 } & 1 & 820 & 33.66 & 829 & 22.56 & 227 & 9.09 \\
\hline 0.45 & 1 & 820 & 33.66 & & & & \\
\cline { 2 - 6 } & 2 & 477 & 25.20 & 438 & 17.30 & & \\
\hline
\end{tabular}

Table 6 Failure deflections under ambient temperature predicted by the full and simplified models

\begin{tabular}{|c|c|c|}
\hline & CM(approach 1) & IM \\
\hline full & $694 \mathrm{~mm}$ & $787 \mathrm{~mm}$ \\
\hline simplified & $699 \mathrm{~mm}$ & $791 \mathrm{~mm}$ \\
\hline
\end{tabular}


Table 7 Geometric and material properties of the slab under elevated temperatures

\begin{tabular}{|c|c|c|c|c|c|}
\hline$a$ & $9 m$ & $\Delta T_{r}$ & $400^{0} \mathrm{C}$ & $T_{y}$ & $135 \mathrm{~N} / \mathrm{mm}$ \\
\hline$b$ & $6 m$ & $\Delta T_{t b}$ & $600^{0} \mathrm{C}$ & $T_{u}$ & $213.75 \mathrm{~N} / \mathrm{mm}$ \\
\hline$d$ & $75 \mathrm{~mm}$ & $E_{2}$ & $1020 \mathrm{MPa}$ & $\alpha_{c}$ & $8 \cdot 10^{-6}$ \\
\hline$h$ & $150 \mathrm{~mm}$ & $A_{s}$ & $0.45 \mathrm{~mm}^{2} / \mathrm{mm}$ & $\alpha_{s}$ & $14 \cdot 10^{-6}$ \\
\hline
\end{tabular}

Table 8 Failure deflections under elevated temperatures using the full and the simplified models

\begin{tabular}{|c|c|c|}
\hline & CM(approach 1) & IM \\
\hline full & $920 \mathrm{~mm}$ & $1251 \mathrm{~mm}$ \\
\hline simplified & $922 \mathrm{~mm}$ & $1266 \mathrm{~mm}$ \\
\hline
\end{tabular}




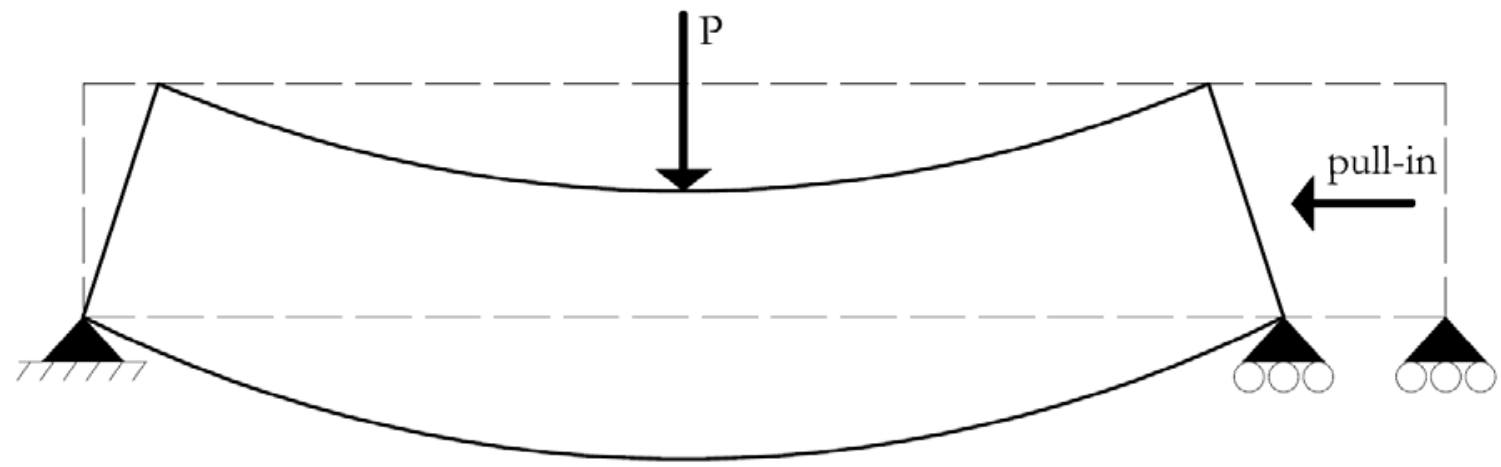

Figure 1: Schematic representation of a beam under large deformations 


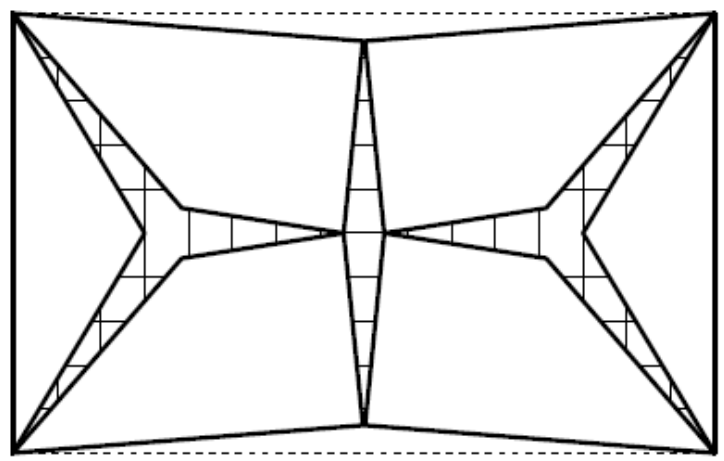

(a) $\mathrm{CM}$

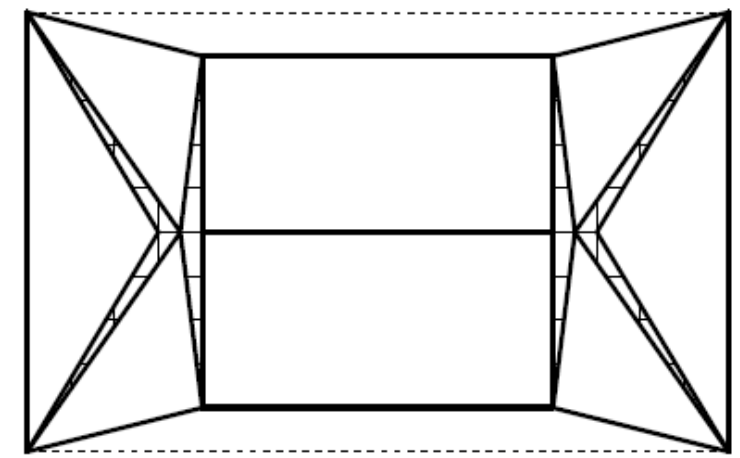

(b) IM

Figure 2: Variants of unrestrained slab model with full depth cracks across the short span at different locations 


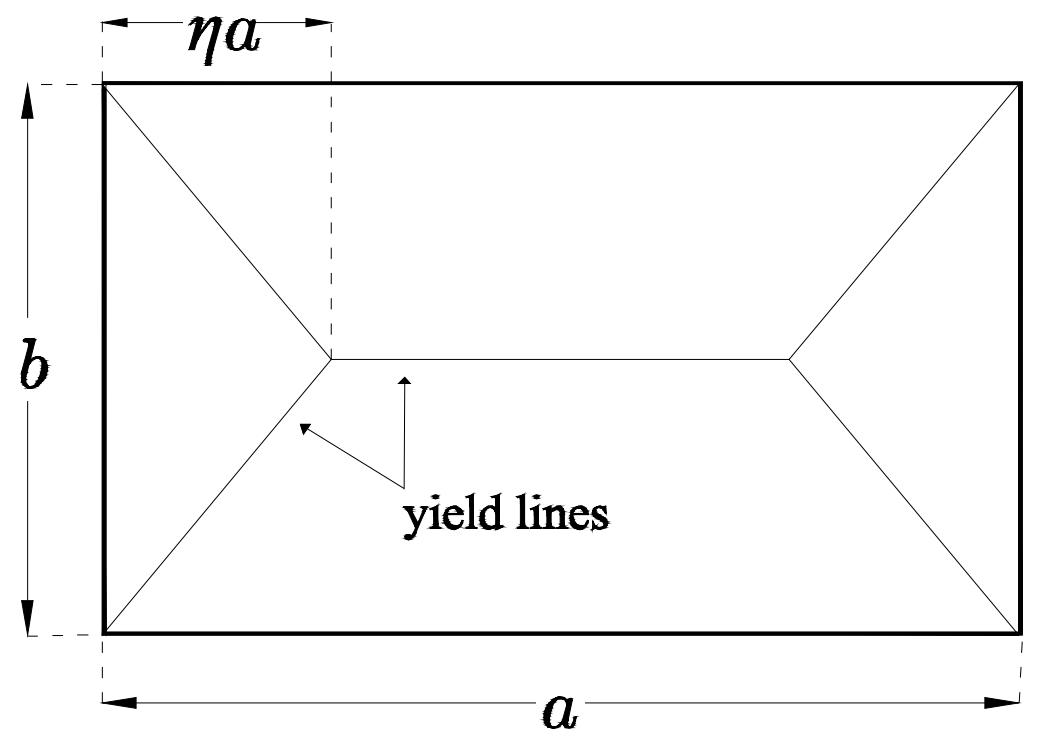

Figure 3: Yield lines forming in a slab 

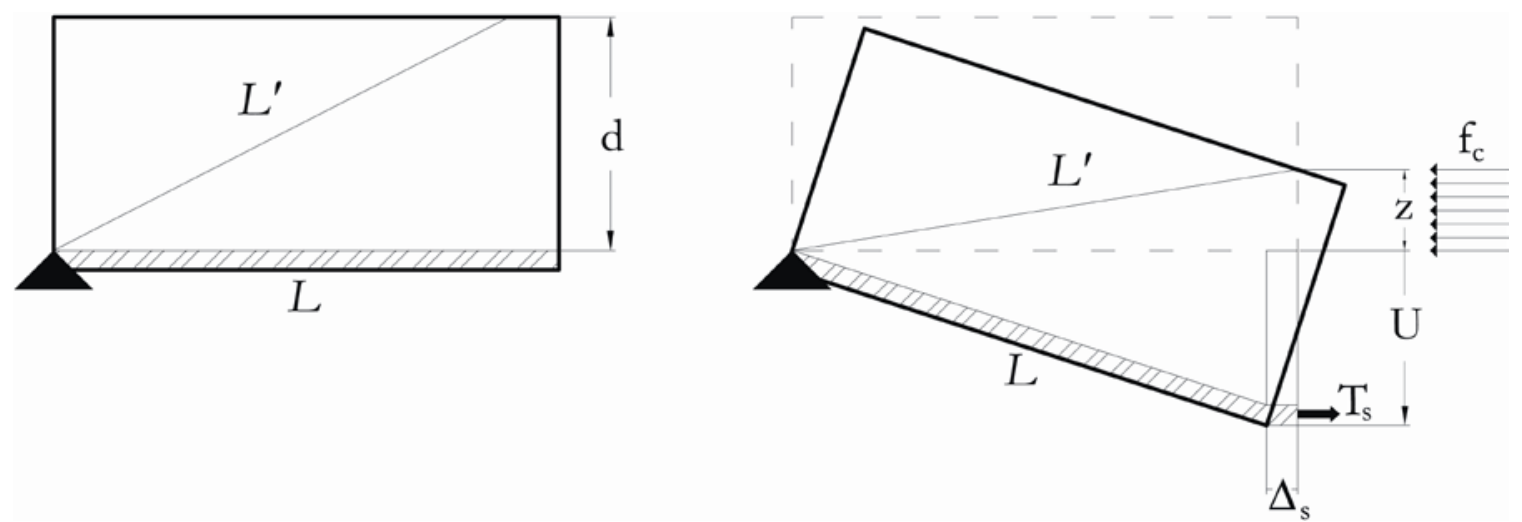

Figure 4: Schematic representation of the deformed beam 


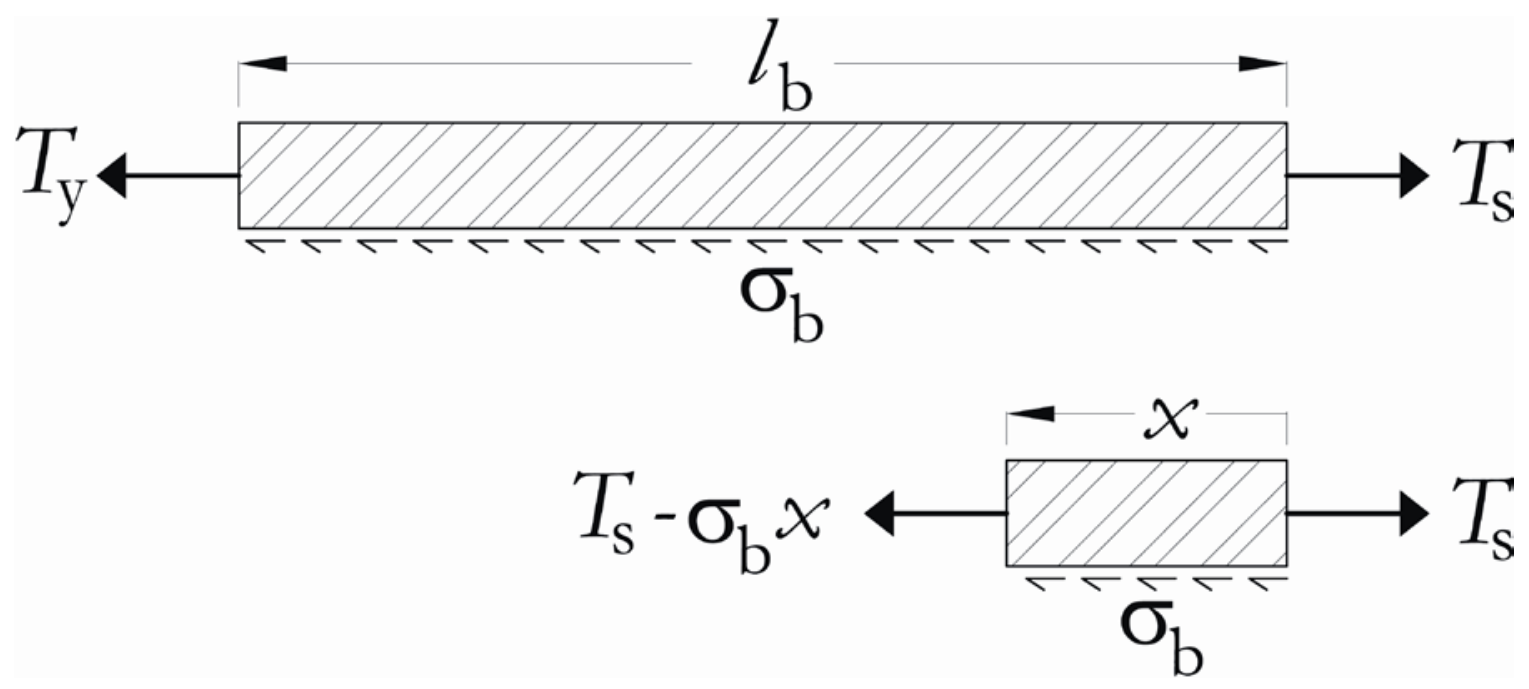

Figure 5: Influence of bond stresses developed along the reinforcement 


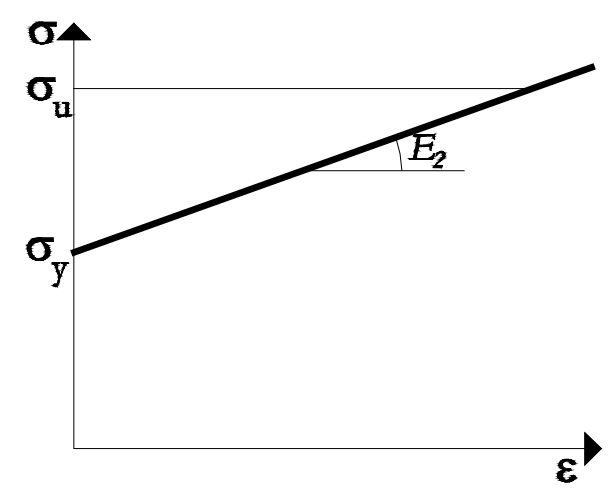

(a)

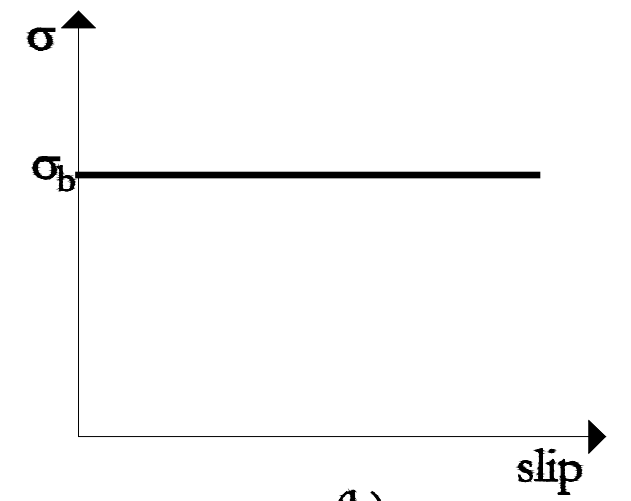

(b)

Figure 6: Material models adopted for a) steel and b) bond strength 


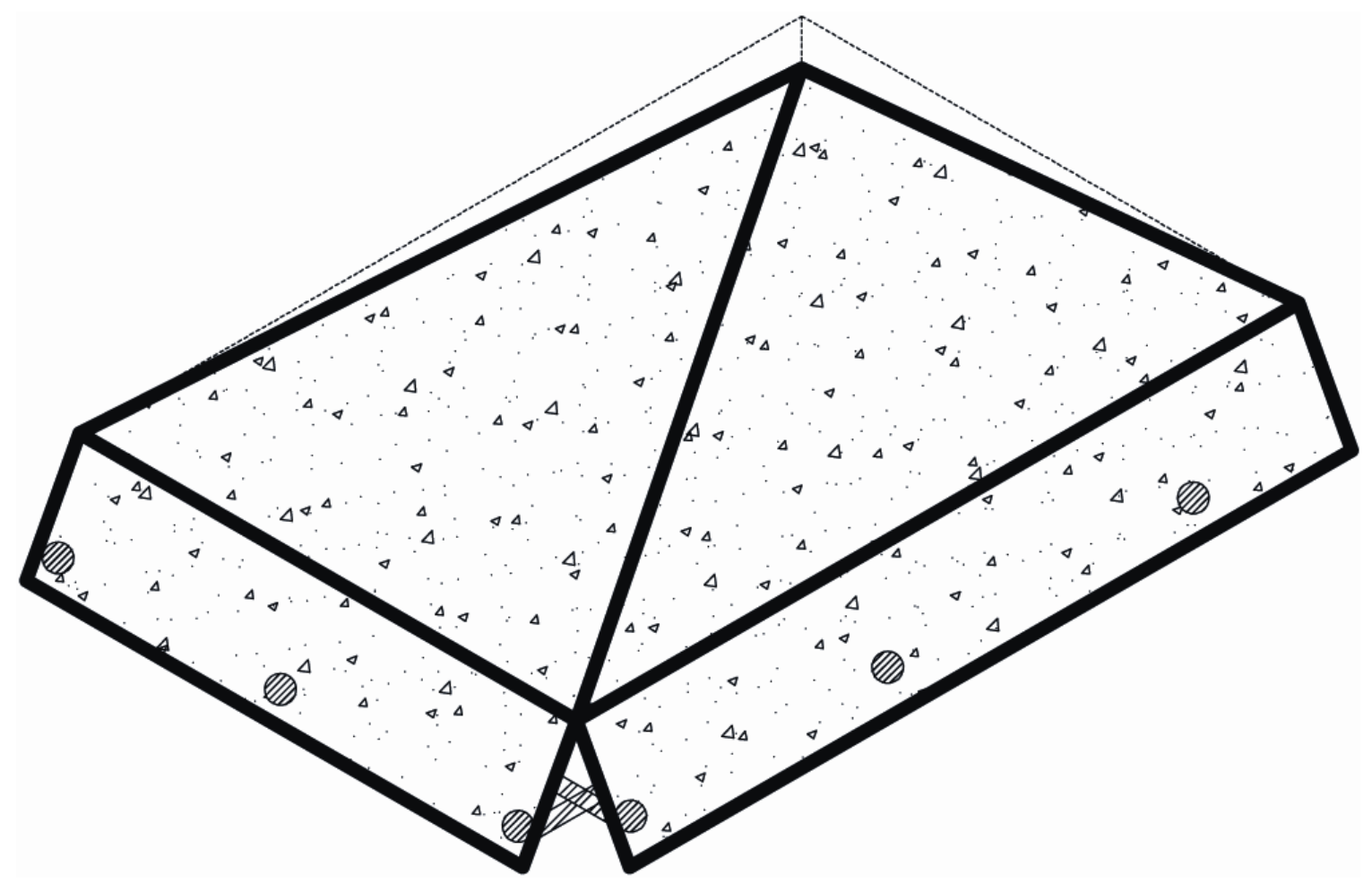

Figure 7: Schematic representation of the contact at the slab corner 


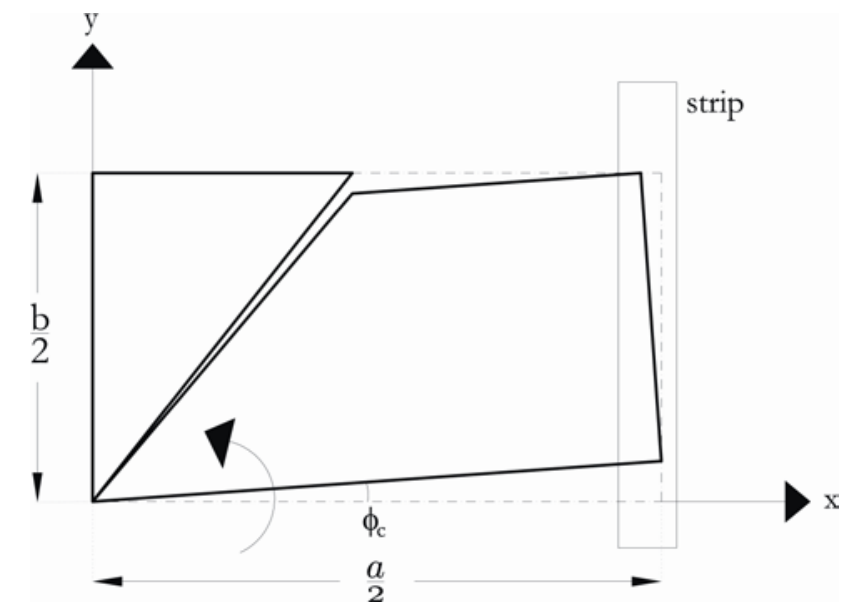

(a) top surface

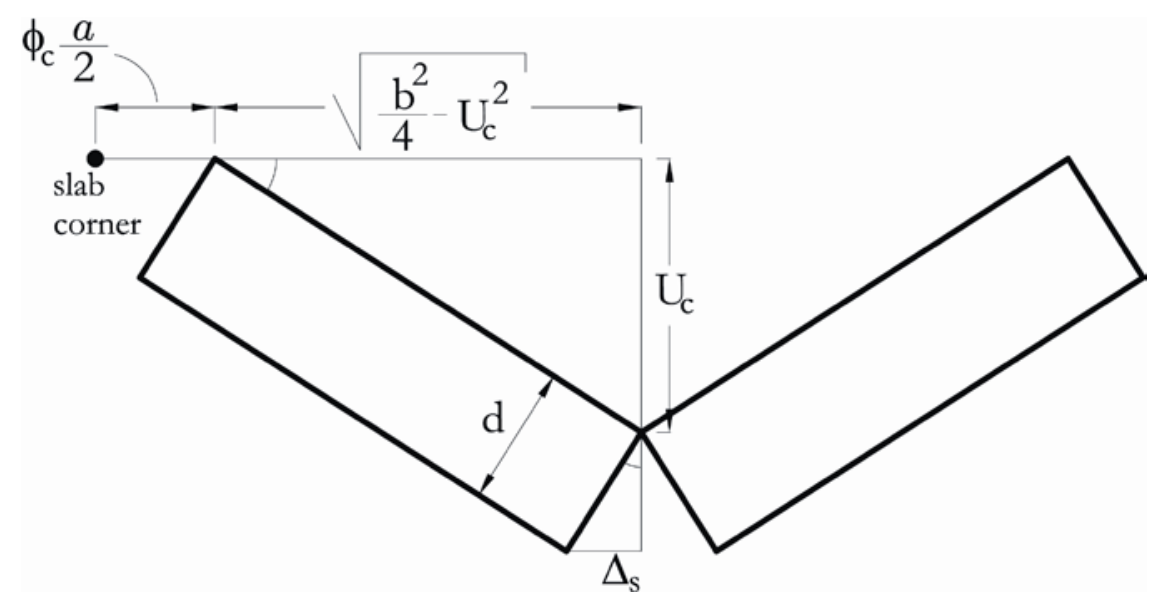

(b) strip

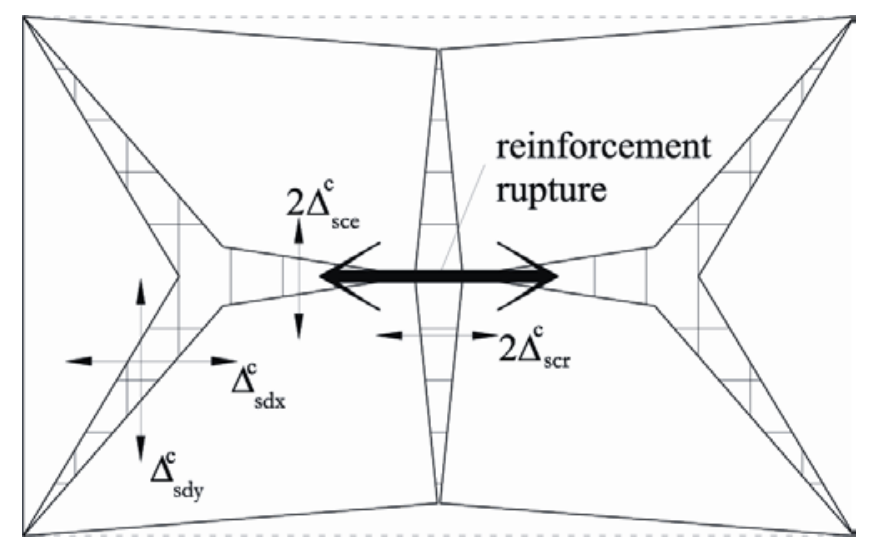

(c) reinforcement extension

Figure 8: Schematic representation of the deflected slab configuration and the reinforcement extension for the CM model 


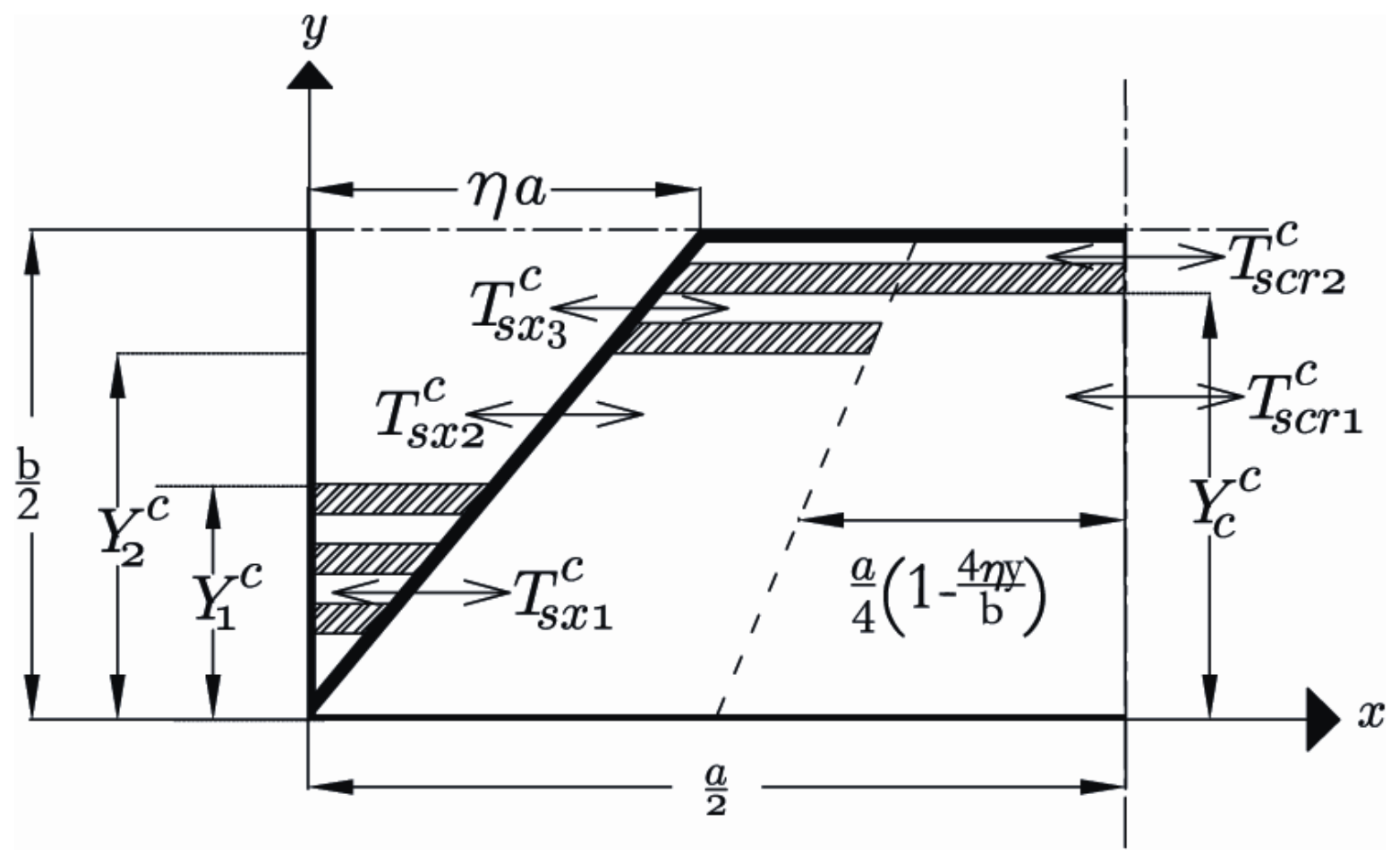

Figure 9: Forces in the reinforcement spanning in the x-direction 

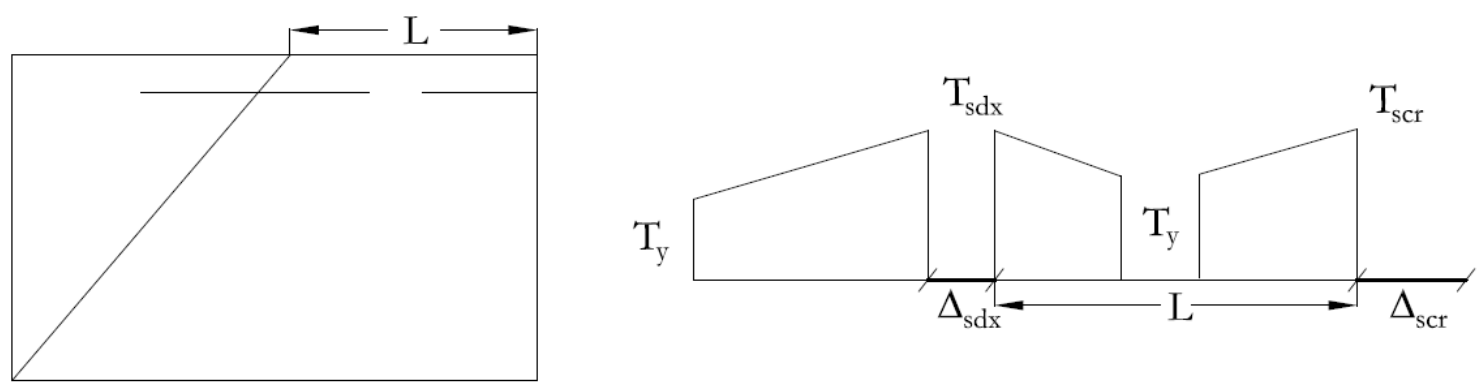

(a) stage 1
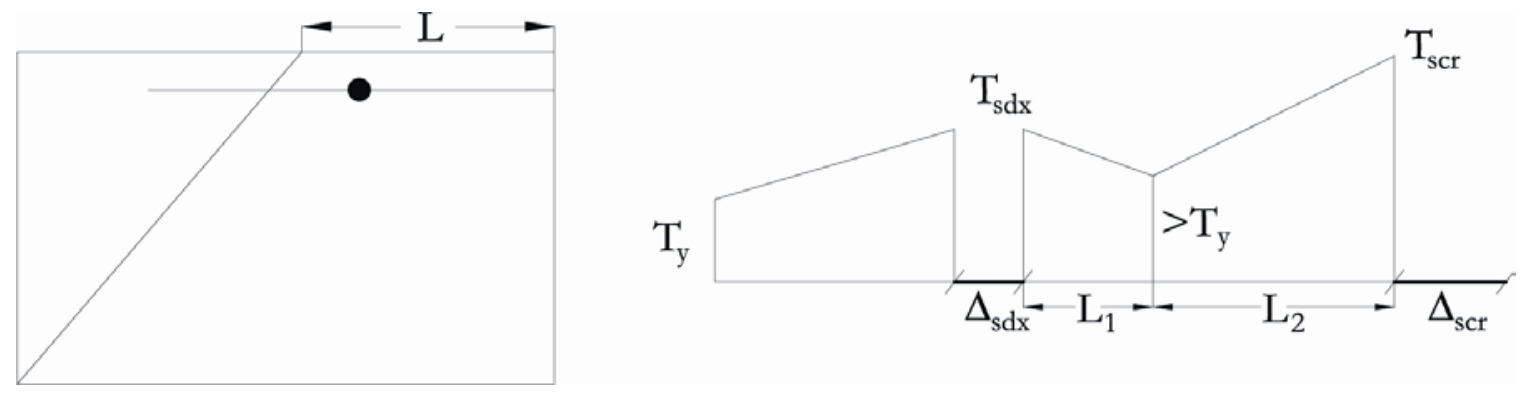

(b) stage 2
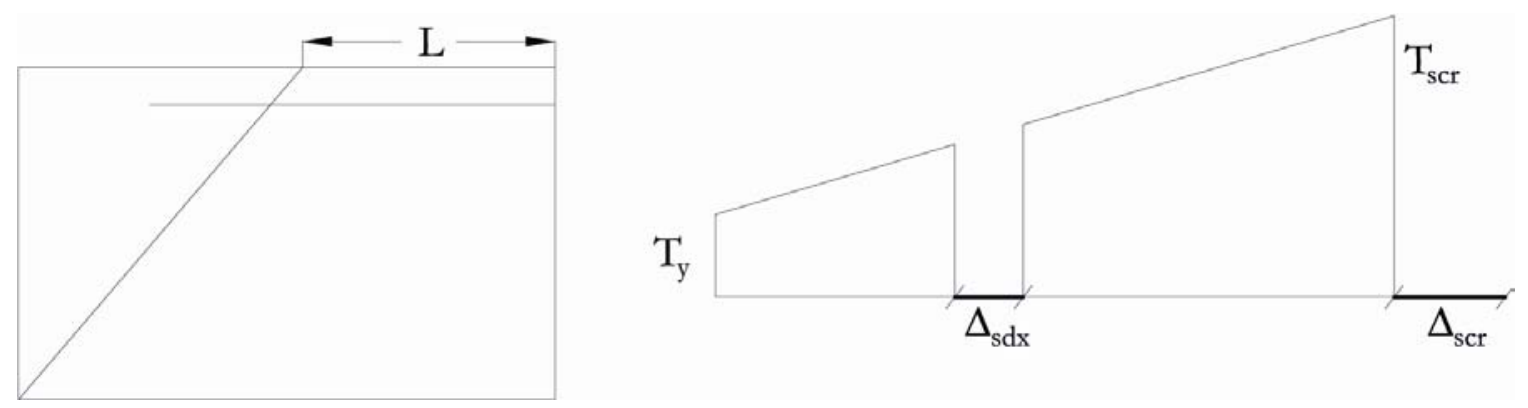

(c) stage 3

Figure 10: Reinforcement force profile in the long direction at various stages of slab response 


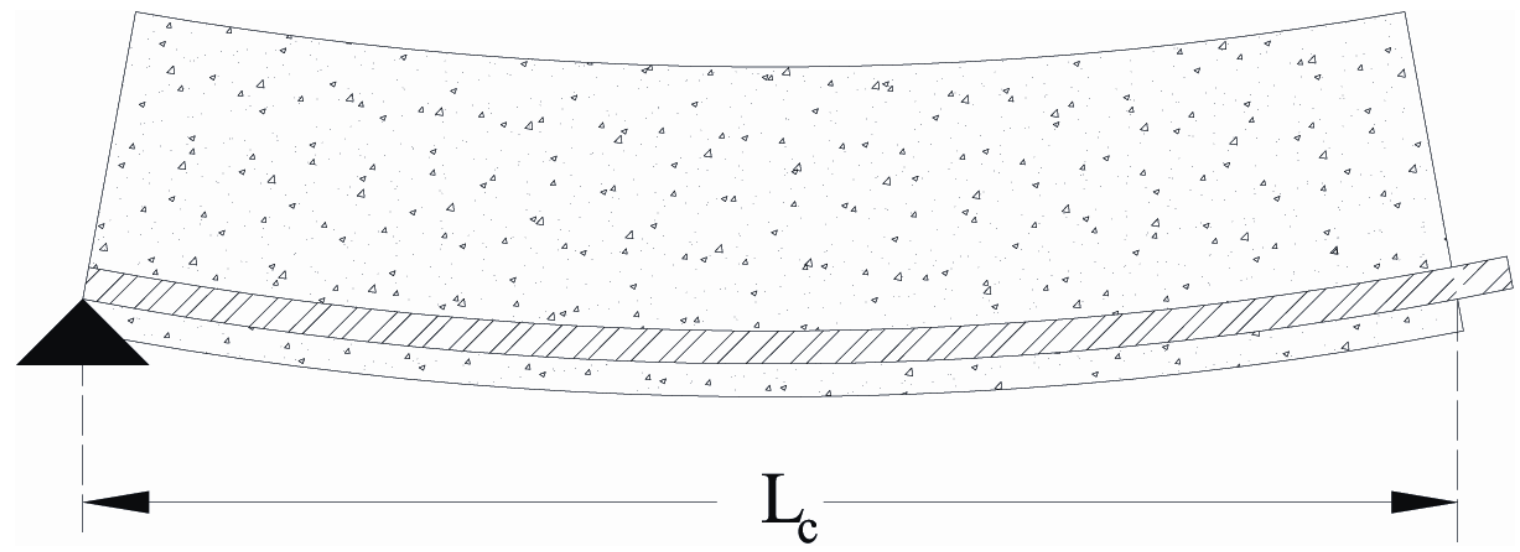

Figure 11: Thermally curved unrestrained half beam strip 


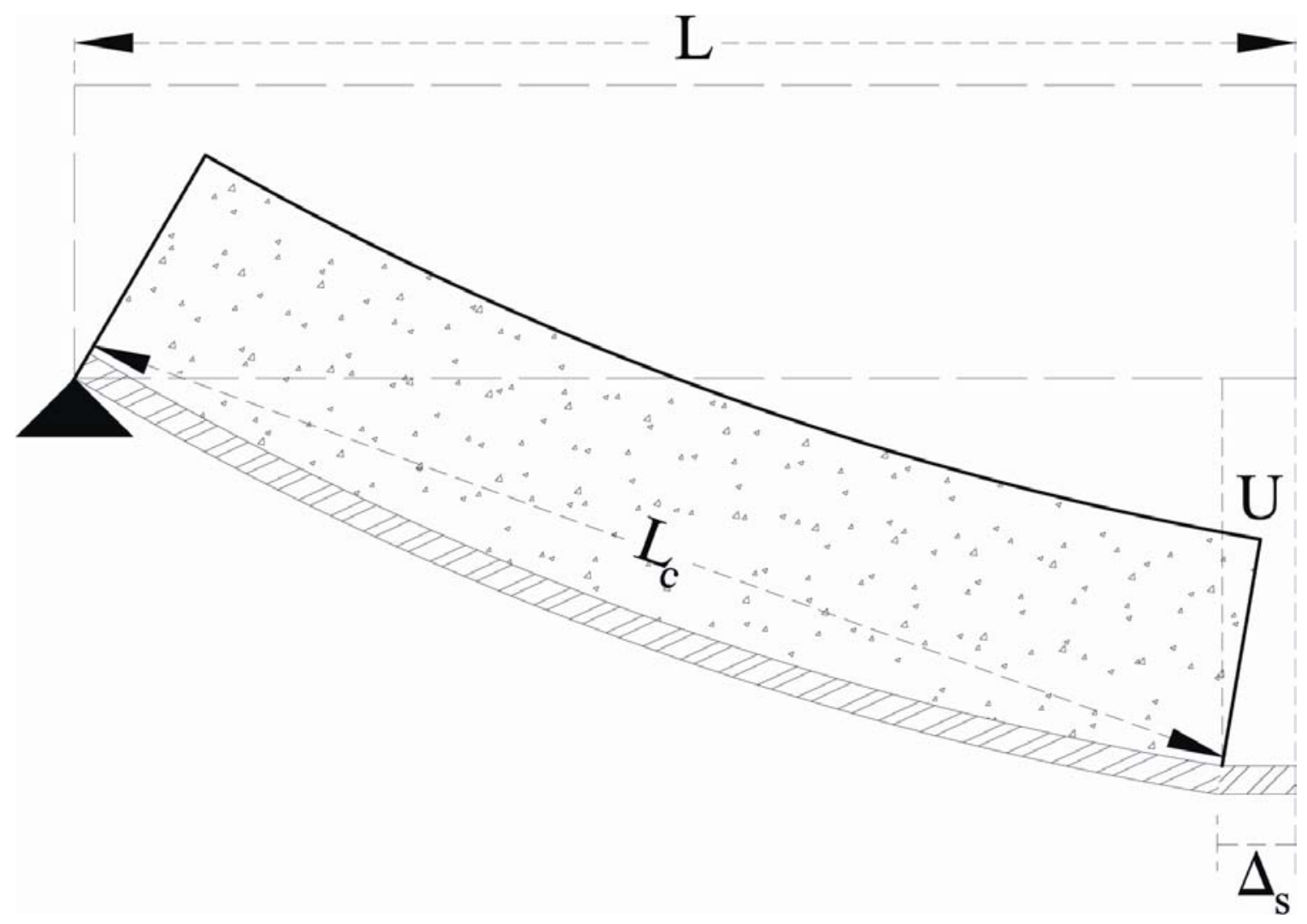

Figure 12: Reinforcement extension for a thermally curved beam strip 


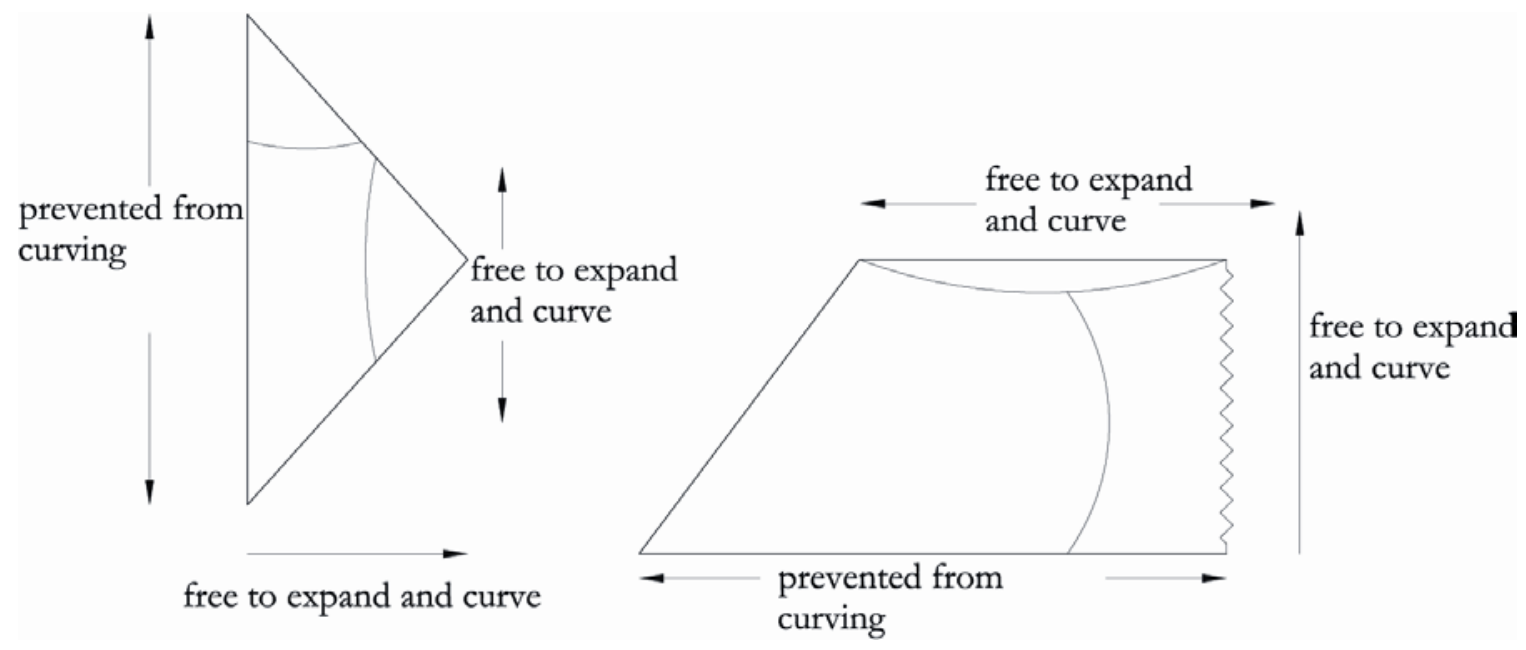

(a)

(a) $\mathrm{CM}$

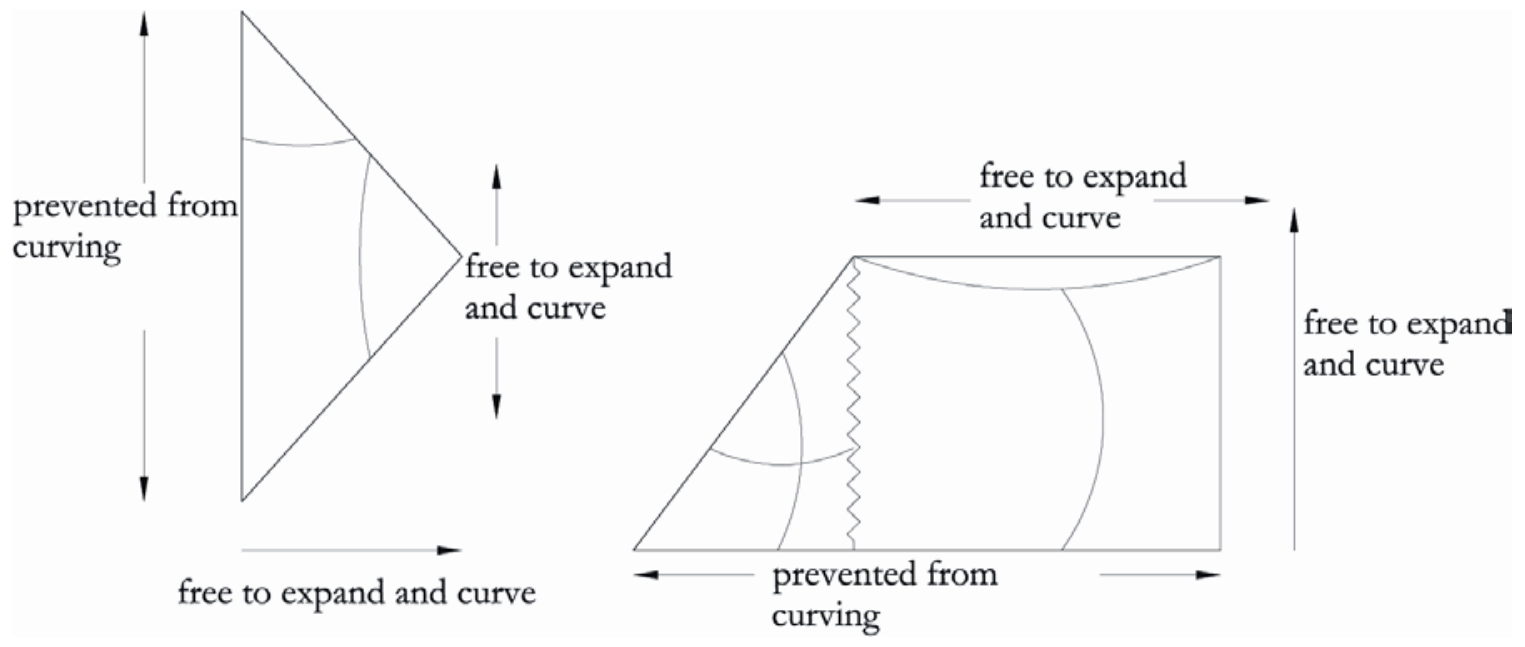

(b) IM

Figure 13: Schematic representation of the thermally curved slab shapes 


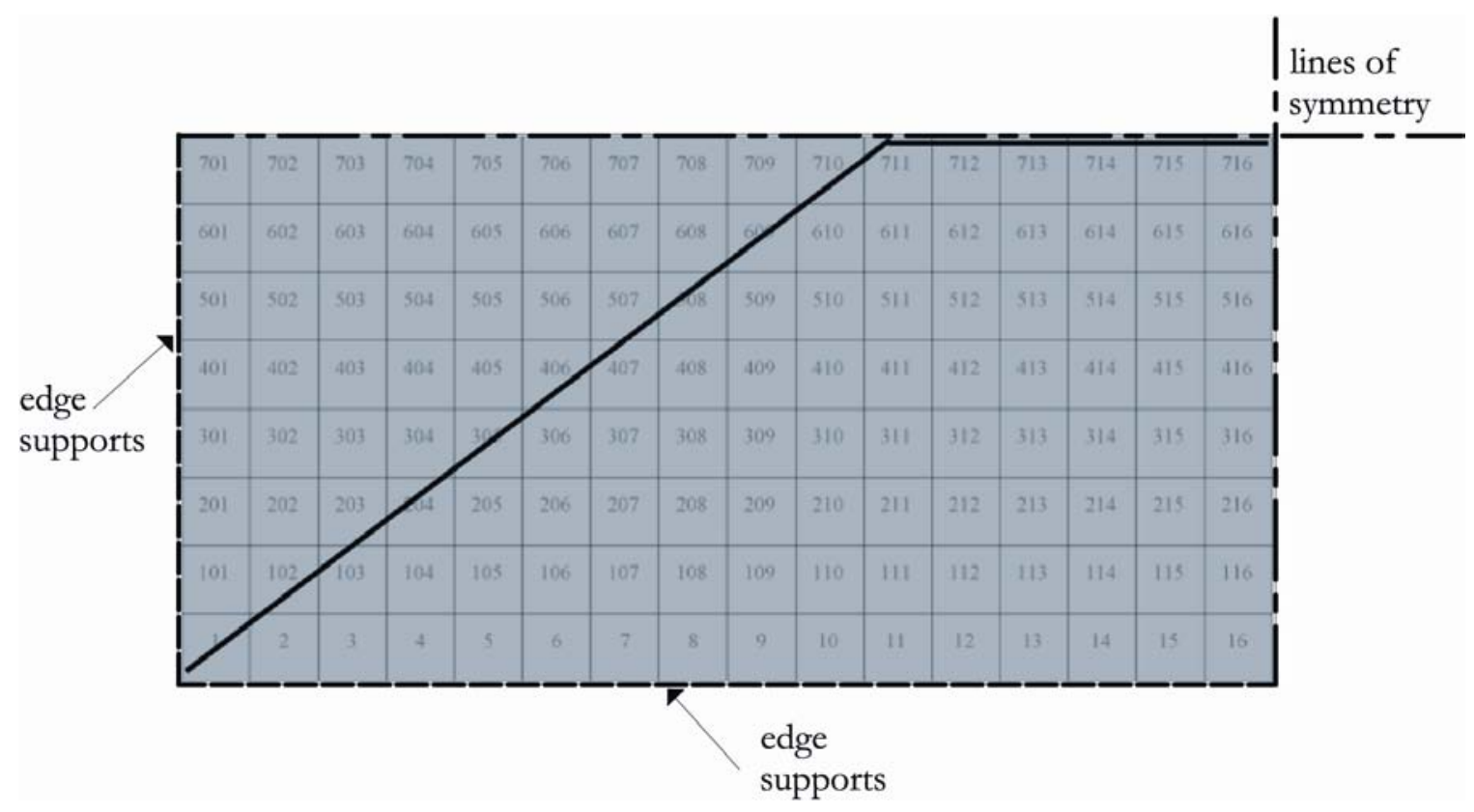

Figure 14: Quarter of the restrained slab analysed with ADAPTIC for $a=12 \mathrm{~m}$ 


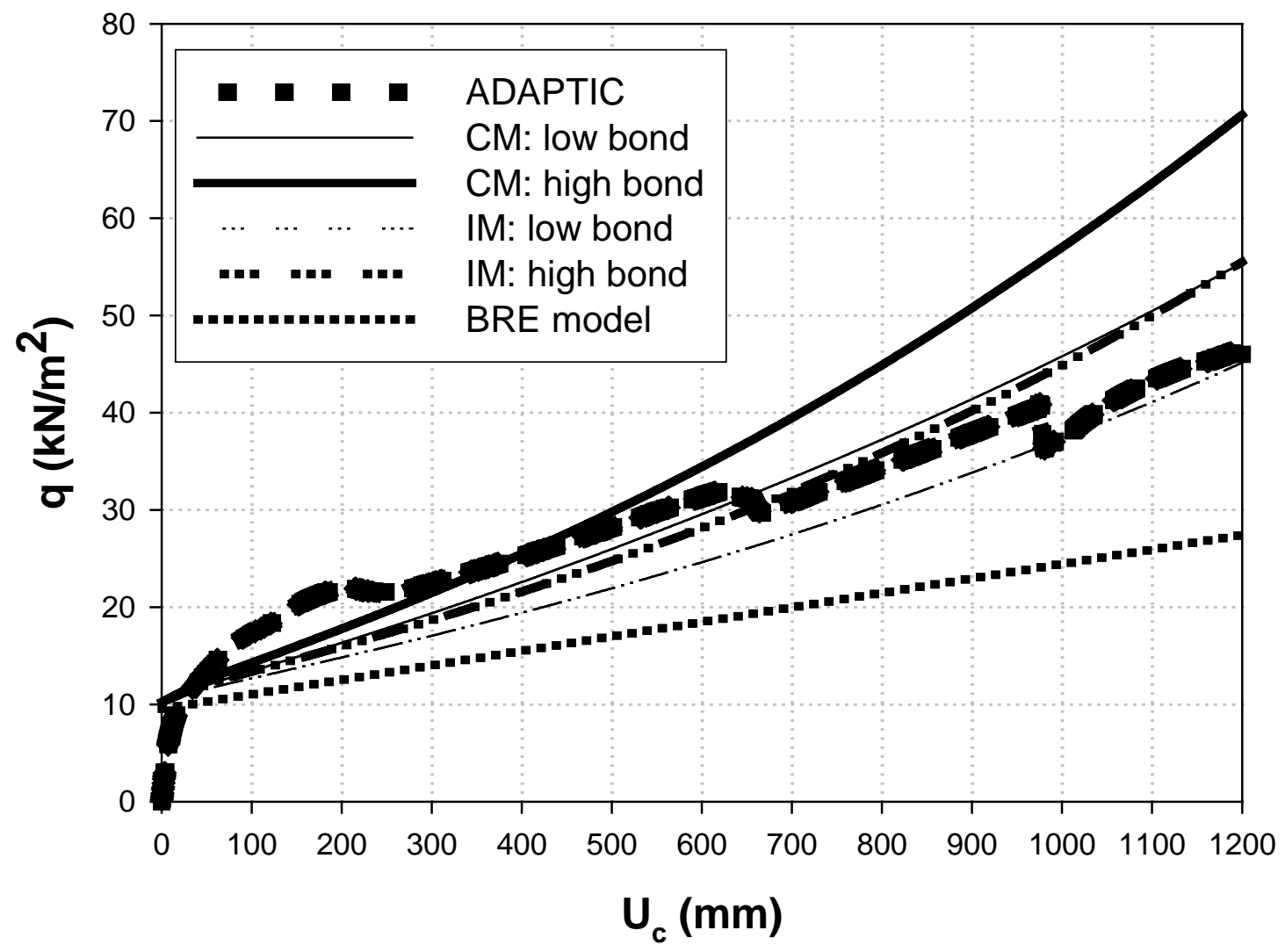

Figure 15: Load deflection response for $a=9 m$ under ambient temperatures 


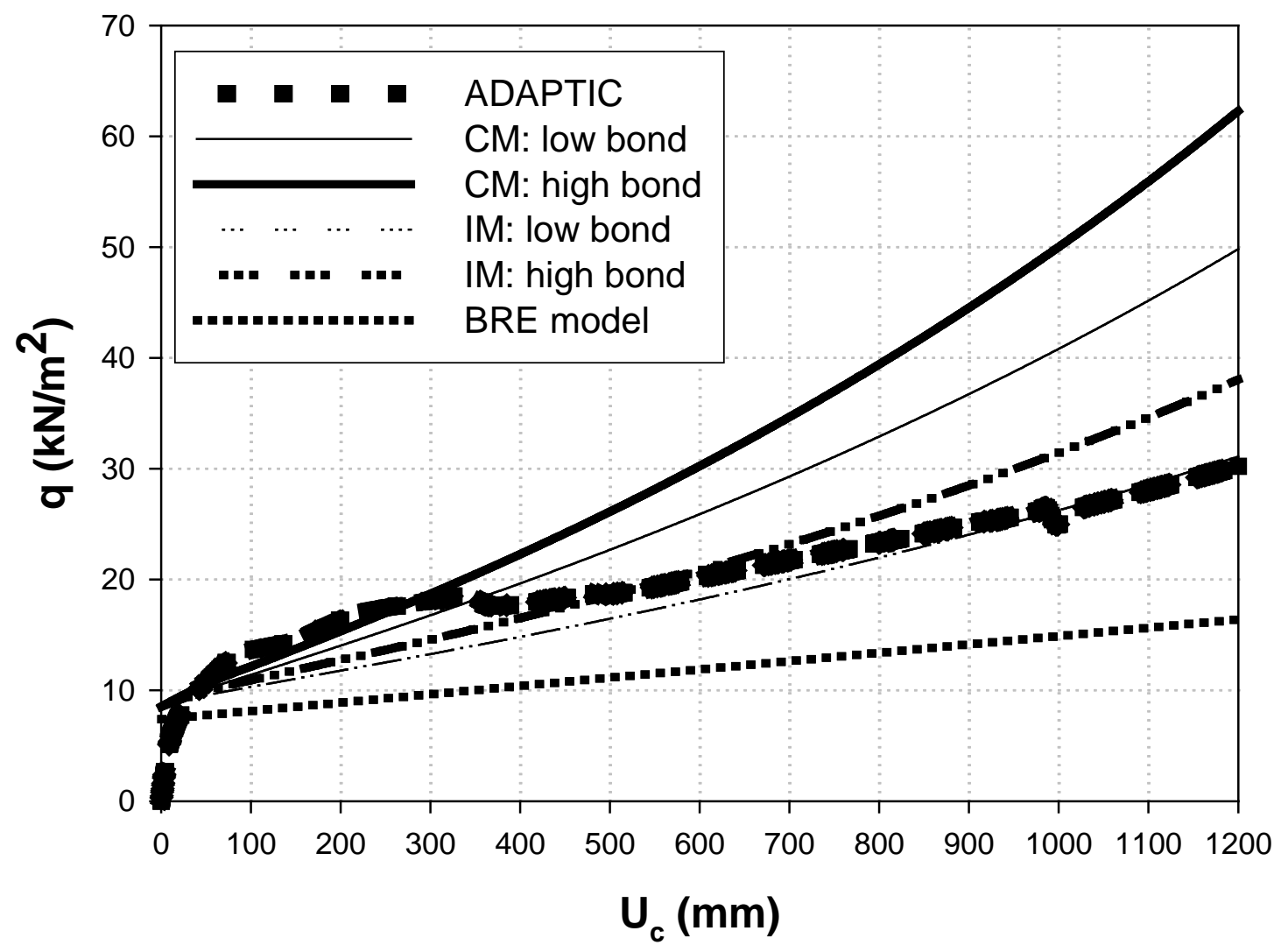

Figure 16: Load deflection response for $a=12 m$ under ambient temperatures 


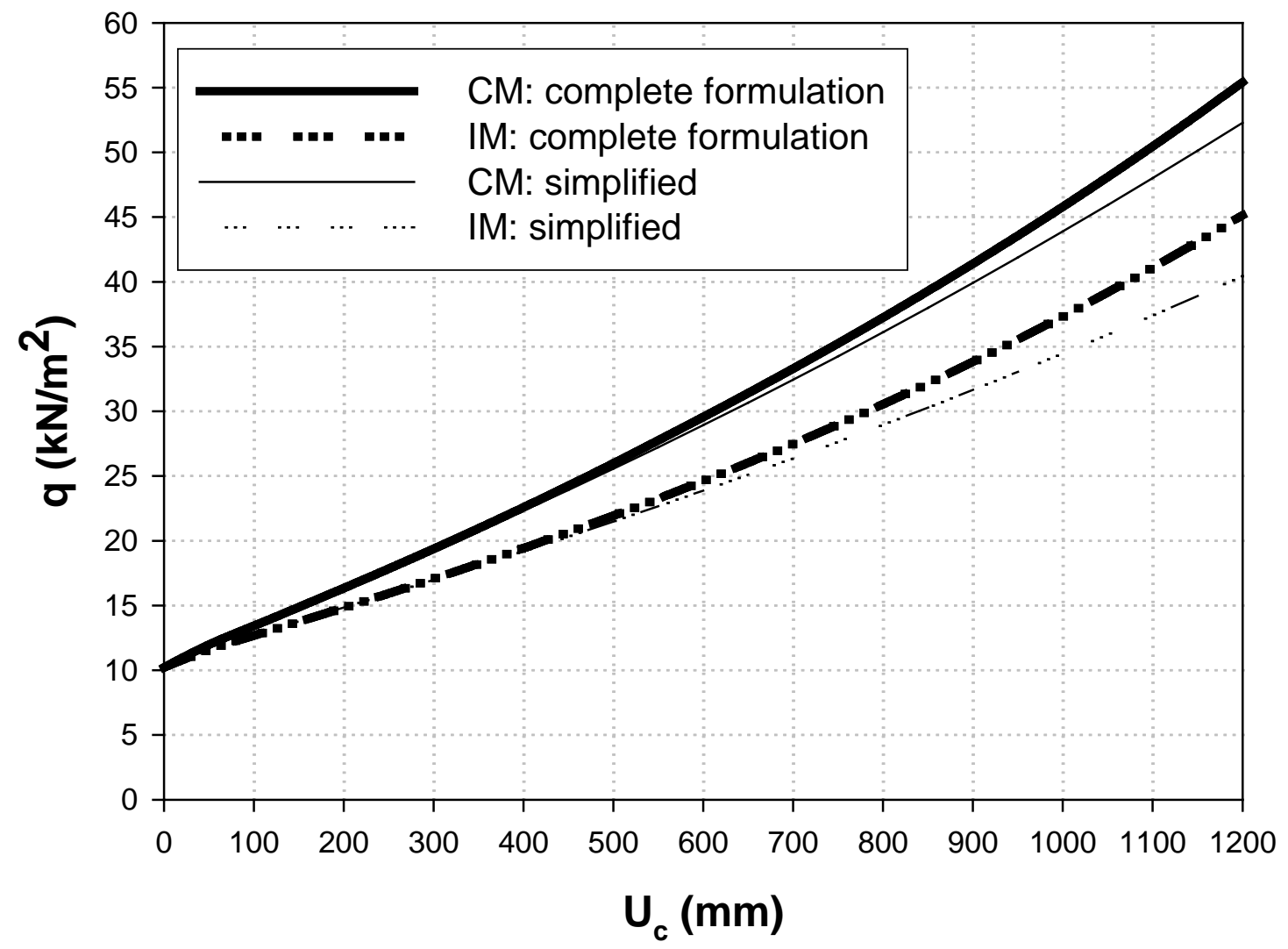

Figure 17: Load deflection response using the complete and simplified approaches under ambient temperature 


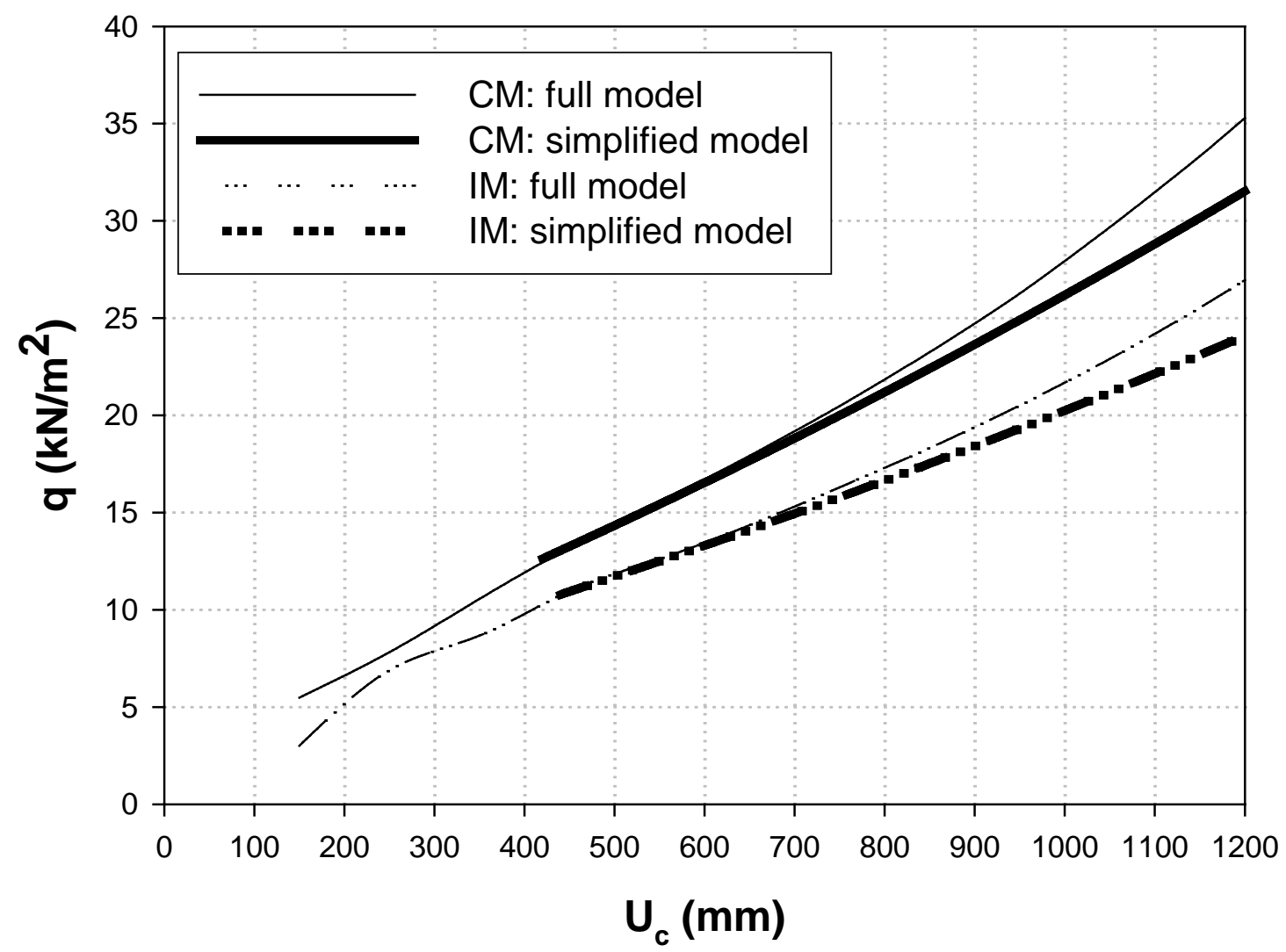

Figure 18: Load deflection response using the full and simplified models under elevated temperature 


\section{References}

[1] A. Y. Elghazouli and B. A. Izzuddin. Response of idealised composite beam-slab systems under fire conditions. Journal of Constructional Steel Research, 56:199-224, 2000.

[2] B. A. Izzuddin and D. B. Moore. Lessons from a full-scale fire test. Proceedings of the Institution of Civil Engineers, Structures and Buildings, 152(3):1-11, 2002.

[3] Z. Huang, I. W. Burgess, and R. J. Plank. Nonlinear analysis of reinforced concrete slabs subjected to fire. American Concrete Institute Structural Journal, 96(1):127-135, 1999.

[4] A. Y. Elghazouli, B. A. Izzuddin, and A. J. Richardson. Numerical modelling of the structural fire behaviour of composite buildings. Fire Safety Journal, 35:279-297, 2000.

[5] C. G. Bailey and D. B. Moore. The structural behaviour of steel frames with composite floorslabs subject to fire: Part1: Theory. The Structural Engineer, 78(11):19-27, 2000.

[6] A. S. Usmani and N. J. K. Cameron. Limit capacity of laterally restrained reinforced concrete floor slabs in fire. Cement \& Concrete Composites, 26:127-140, 2004.

[7] BSI, London. BS 5950 Structural use of steelwork in building: Part 8: Code of practice for fire resistant design, 2003.

[8] R. H. Wood and L. L. Jones. Yield line analysis of slabs. Thames \& Hudson, London, 1967.

[9] L. Lim and C. Wade. Experimental fire tests of two-way concrete slabs. Fire Engineering Research Report 02/12, University of Canterbury, Christchurch, New Zealand, September 2002. ISSN:1173-5996.

[10] E. Omer, B. A. Izzuddin, and A. Y. Elghazouli. Failure of lightly reinforced concrete floor 
slabs with planar edge restraints under fire. Journal of Structural Engineering, ASCE, Vol. 135, No. 9, September 2009, pp. 1068-1080.

[11] B. A. Izzuddin and A. Y. Elghazouli. Failure of lightly reinforced concrete members under fire. I: Analytical modelling. Journal of Structural Engineering, ASCE, 130(1):3-17, January 2004.

[12] E. Omer, B.A. Izzuddin, A.Y. Elghazouli, "Failure of Unrestrained Lightly Reinforced Concrete Slabs Under Fire - Part II: Verification and Application”, (Companion Paper).

[13] A. Sawczuk and L. Winnicki. Plastic behavior of simply supported reinforced concrete plates at moderately large deflections. International Journal of Solids and Structures, 1:97111, 1965.

[14] C. G. Bailey, D. S. White, and D. B. Moore. The tensile membrane action of unrestrained composite slabs simulated under fire conditions. Engineering Structures, 22(12):15831595, 2000.

[15] A. J. Foster, C. G. Bailey, I. W. Burgess, and R. J. Plank. Experimental behaviour of concrete floor slabs at large displacements. Engineering Structures, 26:1231-1247, 2004.

[16] C. G. Bailey. Membrane action of unrestrained lightly reinforced concrete slabs at large displacements. Engineering Structures, 23:470-483, 2001.

[17] E. Omer. Failure of composite steel-concrete slabs under elevated temperatures. PhD thesis, Imperial College, London (http://spiral.imperial.ac.uk/), 2006.

[18] C. R. Calladine. Plasticity for Engineers. Ellis Horwood Limited, West Sussex, 1985.

[19] B. A. Izzuddin. Nonlinear dynamic analysis of framed structures. PhD thesis, Imperial College, London, 1991. 
[20] B. A. Izzuddin. ADAPTIC User Manual, Imperial College, London (http://spiral.imperial.ac.uk/), 2009.

[21] B. A. Izzuddin, X. Y. Tao, and A. Y. Elghazouli. Realistic modelling of composite and reinforced concrete floor slabs under extreme loading. I: Analytical method. Journal of Structural Engineering, ASCE, 130(12):1972-1984, December 2004.

[22] BSI, London. BS 8110 Structural use of concrete: Part 1: Code of practice for design and construction, 1997. 